\title{
das ist ein: (--) äh ja ANschluss ja. Das ja als Turnhalte- und An- schlusssignal im gesprochenen Deutsch.
}

\author{
Dario Marić (Sarajevo)
}

\begin{abstract}
Spoken production is subjected to speed and needs in the conversations that can locally be changed, which makes it dynamic and flexible. Often the thematic framework of the next utterance is determined while the appropriate complex linguistic construction for it is not yet available. Delays and pauses before continuing in the utterances are traces of searching words and an indication that we often decide on the linguistic structure in terms of complex and schematic constructions in the construction grammar before we fill it with vocabulary. This paper is about the functions of organizing the conversations, phonetic and phonological features of a particular $j a$ from within the turn, often the utterance, and the intonation unit in German which at the problems of finding words of speakers mainly mean "immediately resume". In this context, intonation incorporation of this $j a$ into what is uttered just before and immediately after it, is especially indicative. Continuation-ja with independent intonation contour namely announces new construction, while intonation incorporated continuation-ja prevents premature conclusion of the listener that the sentence will be interrupted and announce one or more components of the utterance or correction of specific components.
\end{abstract}

\section{$1 \quad$ Einleitung}

Hört man sich Gespräche, die deutsche MuttersprachlerInnen miteinander führen, an, fällt einem früher oder später die Vorkommenshäufigkeit der Partikel ja in diesen Gesprächen auf. Achtet man ein wenig auf ihre Stellung innerhalb des Redebeitrags und ihre phonetische Erscheinungsform, stellt man schnell fest, dass sie sehr variabel sind. Sie kommt sowohl turninitial als auch turnfinal und turnintern vor, sie kann mit einem sehr gedehnten und pharingalisierten /a:/ oder mit einer Vokalfarbe des extrem gekürzten und behauchten /a:/ gesprochen werden, die in die Vokalfarbe des [0] übergeht, um nur einige ihrer Erscheinungsformen zu nennen. Wenn sie in benachbarte Äußerungen prosodisch nicht integriert ist, kann sie in Kombination mit allen einfachen Tonhöhenmustern vorkommen. Diese Stellungs- und Erscheinungsvariabilität deutet auf ihre Polyfunktionalität hin. Bisher konnte in Analysen tatsächlich die Funktion der Antwort-, Modal-, Grad- und Gesprächspartikel von ja rekonstruiert werden. Innerhalb der Kategorie $j a$ als Gesprächspartikel sind jedoch m. E. zu wenige Unterkategorien ausdifferenziert worden und selbst diese wenigen sind in Grammatiken des Deutschen kaum zu finden, ganz zu schweigen von Lehrwerken für Deutsch als Fremdsprache. 
Einzige Ausnahme unter den Grammatiken stellt in dieser Hinsicht die IDS Grammatik dar (cf. Zifonun/Hoffmann/Strecker 1997: 374-377).

Die Gesprächspartikel ja lässt bekanntlich Anfangs- und Endrand eines Redebeitrags sowie sein Inneres leichter erkennen, was die Steuerung des Sprecherwechsels und das Verfolgen des Gesprächsverlaufs überhaupt fördert. Im Inneren eines Redebeitrags kann es die einzelnen Schritte einer Sprechhandlung einleiten, wie z. B. die direkte Rede, die zur Vergegenwärtigung einer Aussage dient. In diesem Aufsatz geht es um ein ja aus dem Inneren des Redebeitrags, häufig auch der Äußerung und selbst der Intonationsphrase, das bei Wortfindungsproblemen des Sprechers einem vorzeitigen Schließen auf Satzbruch durch den Hörer vorbeugt und im Wesentlichen „gleich geht's weiter mit der angefangenen Äußerung“ bedeutet. Im Folgenden nenne ich dieses ja Anschluss-ja. Es handelt sich hier um eine korpusgestützte Analyse, für welche 100 konversationelle Verwendungen des Anschluss-ja aufbereitet wurden.

Im ersten Kapitel dieses Aufsatzes widme ich mich der Multifunktionalität der Partikel ja und versuche sie mit eigenen Beispielen aus authentischen Gesprächen zu veranschaulichen. Im zweiten Kapitel versuche ich mich dem Wesen der Wortfindungsprobleme aus dem Blickwinkel der Psycholinguistik und der Konstruktionsgrammatik anzunähern. Im dritten Kapitel gehe ich konversationsanalytisch auf den Sprecherwechselmechanismus in Gesprächen, insbesondere auf die Funktion des Turnhaltens ein. Das vierte Kapitel beinhaltet die Ergebnisse meiner Korpusanalyse, die vor allem Erscheinungsformen und gesprächssteuernde Funktionen des Anschluss-ja sowie die Art der an dieses ja angeschlossenen Konstruktionskonstituenten betreffen wird.

\section{$2 \quad$ Multifunktionalität des $j a$}

Folgende Darstellung erhebt keinen Anspruch auf Auflistung aller Funktionen des $j a$ sondern soll vor allem dazu verhelfen, eine Definition des Anschluss-ja auszuarbeiten.

\subsection{Turninitiale Einbettung}

\subsubsection{Ja als Antwortpartikel}

Die Antwortpartikel ja kann sowohl turninitial als auch turnintern und turnfinal eingesetzt werden, wobei der turninitiale Gebrauch mit Abstand der häufigste ist. Funktional ist sie imstande, eine durch eine gestellte Frage entstandene Erwiderungserwartung (cf. 2conditional relevance“, Schegloff 1968: 1083) allein zu erfüllen. Ein im Verberstfragesatz geäußertes Unwissen oder eine Unsicherheit über die Geltung eines Sachverhalts wird nämlich dadurch beseitigt, dass die Geltung des Sachverhalts bestätigt wird. Die alle semantischen Konstituenten aus dem Fragesatz enthaltende Proposition, die die Geltung des Sachverhalts bestätigt, kann dem ja redundanterweise nachgestellt werden.

Beispiel für eine Antwortpartikel stammt aus einer Radio-Phone-In-Sendung zum Thema Integration der Schüler mit einer körperlichen oder geistigen Behinderung in die Regelschulen:

Transkript 1: ,inklusives Bildungssystem“

10 An: <<dim>das 'GEHT doch gar net.> 


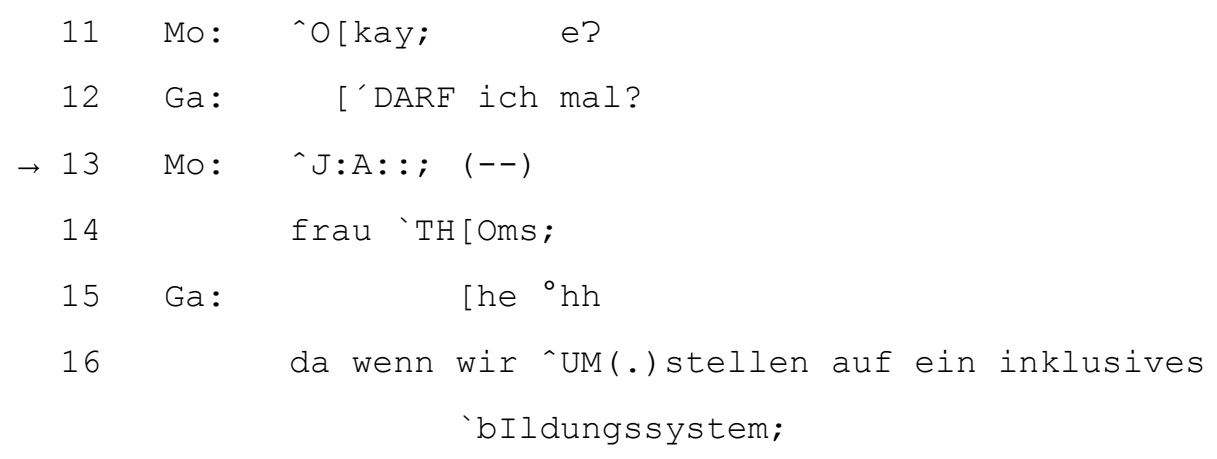

Der Anrufer, der vom Beruf Ergotherapeut in einer Förderschule ist, zweifelt am Erfolg einer solchen Integration. Er problematisiert unmittelbar vor diesem Gesprächsausschnitt den Übergang von der bisherigen Betreuung des behinderten Kindes durch ein Expertenteam der Sonderschulen zur Betreuung des behinderten Kindes auf den einzelnen Lehrer der Regelschulen. Einer der Gäste der Sendung bittet um die Erlaubnis zur Stellungnahme zum Zweifel des Anrufers an der Inklusion (Z. 12). Sequenzorganisatorisch handelt es sich hier um eine Vormitteilung. Die Moderatorin gibt dem Gast die Erlaubnis zur Stellungnahme (Z. 13 und 14), worauf er einen längeren Redebeitrag produziert.

\subsubsection{Ja als Startsignal}

Das $j a$, das das Vorvorfeld der ersten Äußerung im Redebeitrag besetzt, scheint besonders häufig in Redebeiträgen der Sprecher vorzukommen, die eben vom letzten Sprecher z. B. durch Adressieren zum nächsten Sprecher gewählt werden (für das Adressieren cf. Sacks et al. 1974: 704). Nachdem sich der Sprecher selbst zum Sprecher gewählt hat (eigenmächtig das Wort ergriffen hat), leitet der Diskursmarker ja gehäuft Widersprüche ein (cf. Meer 2009: 109-111).

Das Beispiel für das Startsignal $j a$ ist einer Radio-Phone-In-Sendung zum Thema Lohnuntergrenze in Deutschland entnommen:

Transkript 2: „Lohnuntergrenze“

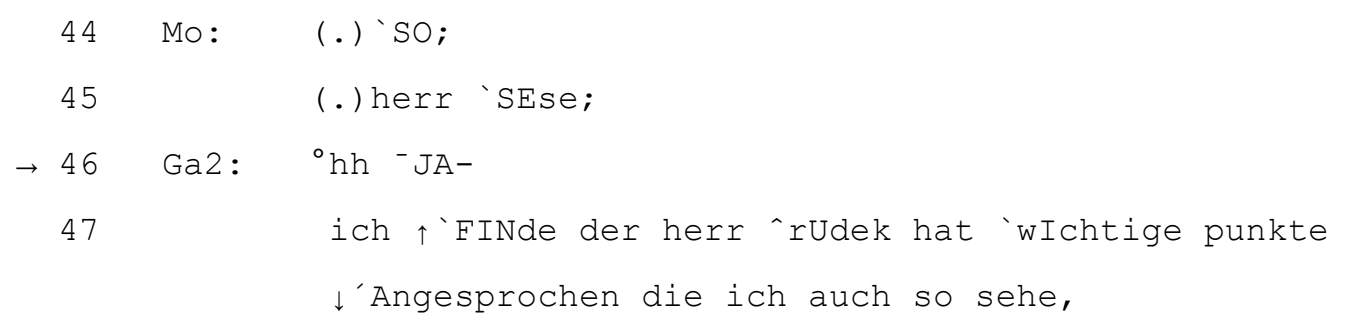

Die Moderatorin beendet in Zeile 44 die Tadelsequenz eines der Gäste, bevor sie in Zeile 45 dem anderen Gast der Sendung das Rederecht erteilt. Er ergreift das Wort (Z. 46), äußert sich lobend über den Redebeitrag des letzten Anrufers und stimmt ihm zu (Z. 47).

\subsection{Turninterne Einbettung}

\subsection{1 $J a$ als Modalpartikel}

Die Modalpartikel ja fügt dem Rest der Äußerung hinzu, dass die Proposition der Äußerung zum geteilten Wissen der Gesprächsbeiteiligten gehört. Das ja in der Funktion einer 
Modalpartikel wird veranschaulicht in einem Gesprächsauschnitt aus einer Radio-Phone-InSendung zum Thema Rolle der Medien beim politischen Absturz des damaligen Bundespräsidenten Christian Wulff. Gäste der Sendung sind ein Politikwissenschaftler und ein Medienwissenschaftler:

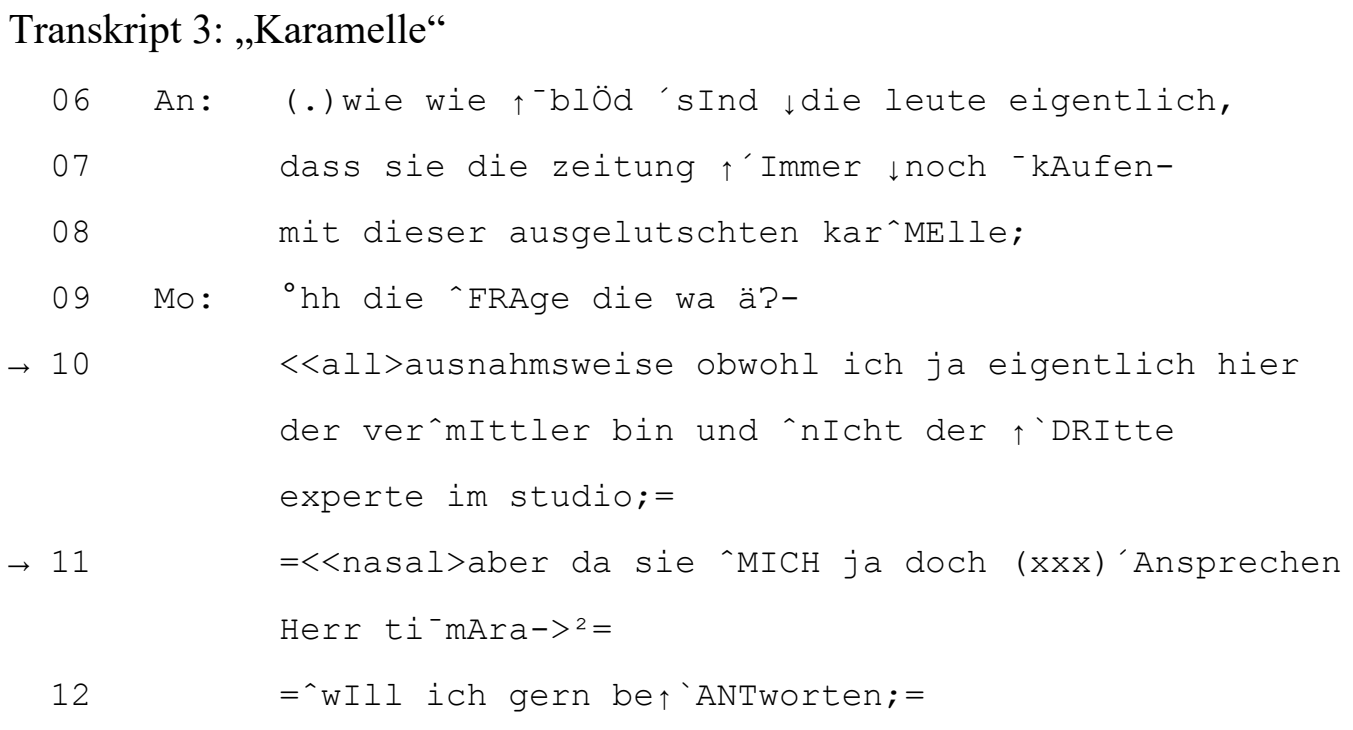

Die Intonationsphrasen (jeweils in Zeilen) 06, 07 und 08 stellen eine Äußerung zusammen, die den Zeitungsjournalisten vorwirft, über die Wulff-Affäre über einen relativ langen Zeitraum hinweg kontinuierlich geschrieben, und dadurch die Wünsche der Leserschaft gar nicht beachtet $\mathrm{zu}$ haben. Diese Äußerung erfragt außerdem leicht die Gründe dieses Verhaltens der Zeitungsjournalisten. Die Frage ist an die Journalisten im Studio und den Moderator selbst gerichtet. Der Moderator übernimmt die Rolle des in diesem Fall Antwort gebenden Gesprächsbeteiligten und nennt zunächst Gründe dagegen obwohl ich ja eigentlich hier der Vermittler bin und nicht der dritte Experte im Studio und danach Gründe dafür Aber da Sie mich ja ansprechen Herr Timara. Die Modalpartikel ja in Zeile 10 bzw. Zeile 11 bezeichnen, dass der Sachverhalt: Der Sprecher ist Moderator der Sendung bzw. der Sprecher ist unter Anderen als Antwortgebender gewählt zum geteilten Wissen der Gesprächsbeteiligten gehört. Am Anfang der Sendung pflegen sich ModeratorenInnen der Radiosendungen nämlich als solche vorzustellen und bestätigen dies immerfort dadurch, dass sie die Sendung moderieren. Dass Journalisten im Studio in der Vorwurfsfrage adressiert sind, weiß man aus Zeilen 02 und 03: Sie mit Ihren Kollegen, auch von den betreffenden Magazinen und Zeitungen.

\subsubsection{Ja als Gradpartikel}

Die Gradpartikel ja leitet turnintern eine Selbstkorrektur im Sinne einer graduellen Steigerung einer unmittelbar davor ausgedrückten Eigenschaft, eines Umstands oder einer Zuordnung zu einer Sammelbezeichnung ein. Wie das Beispiel aus dem letzten Unterkapitel stammt das Beispiel für eine Verwendung der Gradpartikel ja aus einer Radio-Phone-In-Sendung zum Thema Rolle der Medien beim politischen Absturz des damaligen Bundespräsidenten Christian Wulff:

Transkript 4: „Bildzeitung“

01 Ga: ${ }^{\circ}$ hh die 'blldzeitung ${ }^{\circ} \mathrm{h}$ ^kAnn sich im moment 


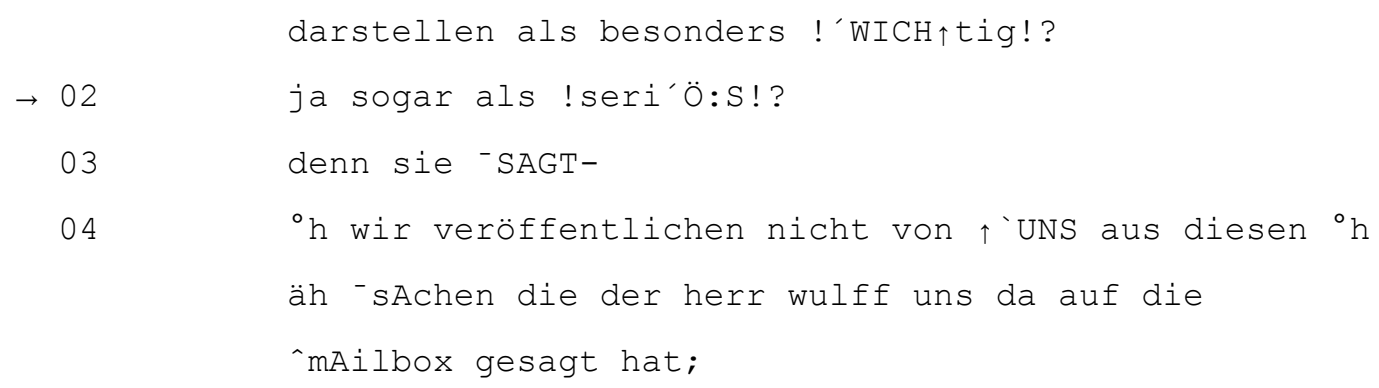

Der Gast im Studio, ein Medienwissenschaftler, kritisiert in diesem Gesprächsausschnitt die Heuchelei der sensationshungrigen Bild-Zeitung in der Wulff-Affäre, die die Schuld für die Affäre abstreitet, obwohl sie gegen Wulff ermittelt und berichtet hat. Die graduelle Steigerung, die hier durch die beiden Gradpartikel ja und sogar zum Ausdruck gebracht wird, betrifft das geheuchelte Wichtigsein der Bildzeitung (Z. 01), das durch die Eigenschaft der Seriosität verstärkt wird (Z. 02).

\subsection{3 ja als Marker der direkten Rede}

Das ja wird außerdem relativ häufig der direkten Rede, die im Gespräch einer Illustration dient, vorangestellt. Im folgenden Gesprächsausschnitt aus einer Radio-Phone-In-Sendung zum Thema Verfassung für das WWW leitet das ja eine mögliche Äußerung eines Messenger- bzw. E-Mail-Nutzers ein:

Transkript 5: „Messenger und E-Mail““

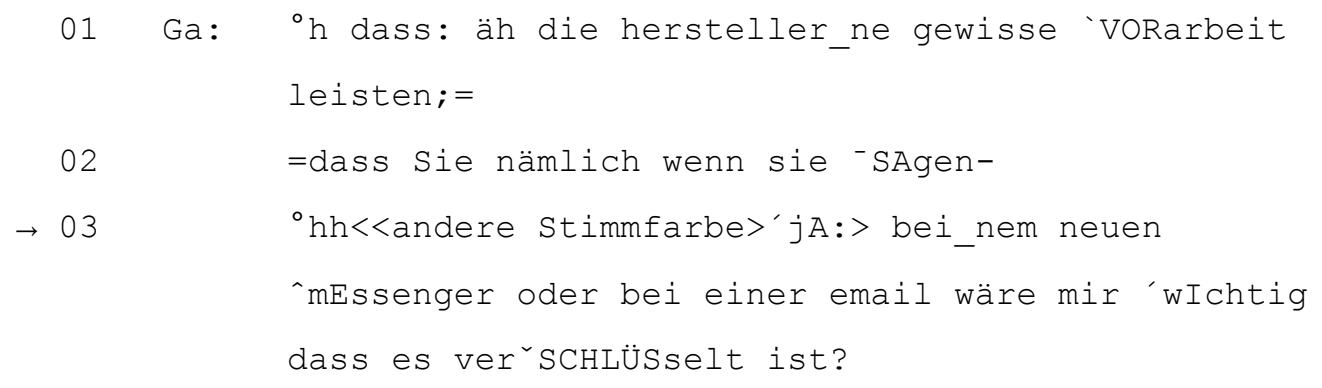

Die in die Sendung eingeladene Expertin für Sicherheit im Internet, befürwortet eine Verschlüsselung der Meldungen bei Messenger- und E-Mail-Diensten durch die Anbieter. Dies könnte ihres Erachtens zur Sicherheit im Internet beitragen. Mit der potentiellen Äußerung eines Messenger- bzw. E-Mail-Nutzers in der direkten Rede spielt sie verstärkt auf das Bewusstsein der Internetnutzer für die Wichtigkeit der Verschlüsselung bei Messenger- und EMail-Diensten an.

\subsubsection{Ja als Tag question}

Der Sprecher kann gelegentlich das ja einer nicht-finalen Äußerung nachstellen, um ihren illokutionären Zweck nachdrücklicher durchzusetzen (cf. Hagemann 2009: 160-170). Dieses ja stellt nämlich die Geltung des propositionalen Gehalts der Vorgängeräußerung als evident dar.

Außerdem kann das ja mit einer steigenden letzten Tonhöhenbewegung ähnlich dem ne, he oder oder (cf. Selting 1995: 272-274) zur Überprüfung eigener Inferenz einer Äußerung nachgestellt sein. Der zweite Teil der Paarsequenz zu diesem ja kann außer dem 
bestätigenden bzw. zurückweisenden Rezipientensignal eine Elaboration bzw. eine Korrektur sein. Das Beispiel für die erstere Verwendung des ja stammt aus der Radio-Phone-InSendung zum Thema Verfassung für das WWW:

Transkript 6: „Diebstahlsicherung“

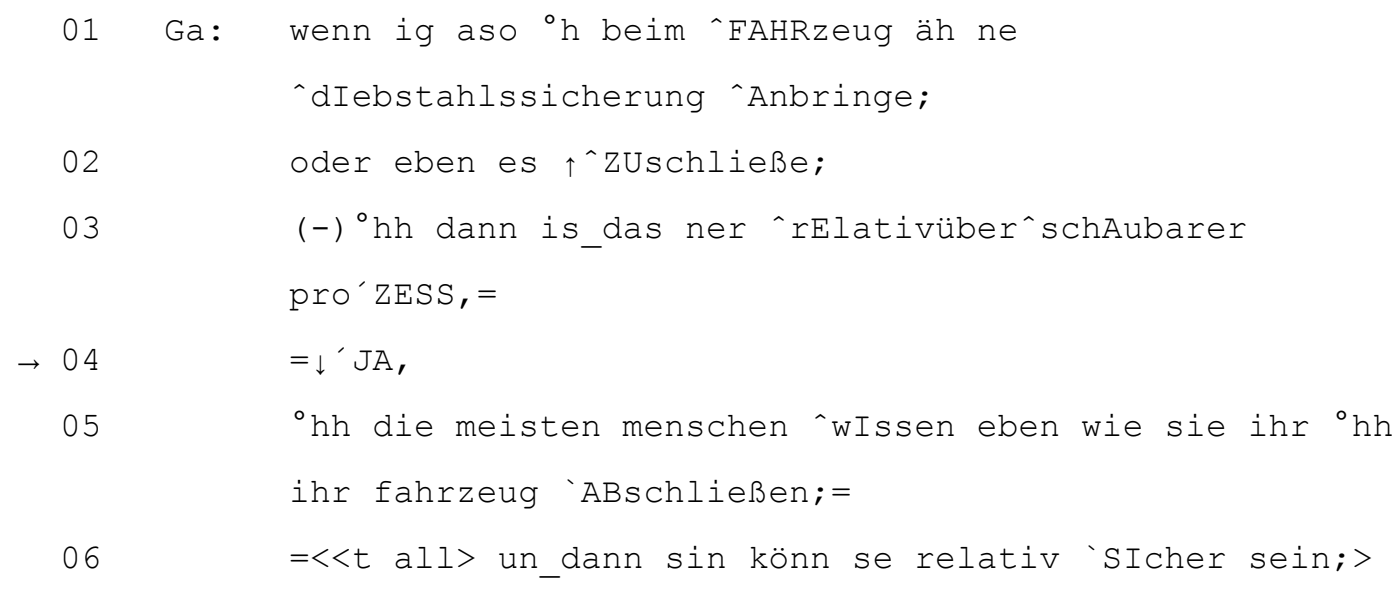

Die Expertin für Sicherheit im Internet plädiert für die Informiertheit der Kunden beim Ankauf von Computertechnik. Die Sequenz aus diesem Gesprächsausschnitt stellt einen Vergleich der Benutzung von Produkten für Auto-Diebstahlsicherung mit der Benutzung von Produkten für PC Sicherheit dar, wobei sich Ersteres als viel einfacher erweist. Das ja wird in Zeile 04 angehängt, um nachdrücklicher von der Einfachheit des Ankaufs, der Installierung und der Benutzung von Produkten für Auto-Diebstahlsicherung zu überzeugen.

\subsection{Turnfinale Einbettung}

\subsubsection{Endsignal}

Wenn der Sprecher das Rederecht endgültig vergeben möchte und dem potentiellen nächsten Sprecher keine weiteren Stellen für eine Rederechtübernahme (siehe Kapitel 4.) bieten möchte, kann er turnfinal (oder unmittelbar davor) ein ja realisieren. Beispiel für eine solche Verwendung des ja stammt aus einer Radio-Phone-In-Sendung zum Thema Prostitution:

Transkript 7: „Prostitution“

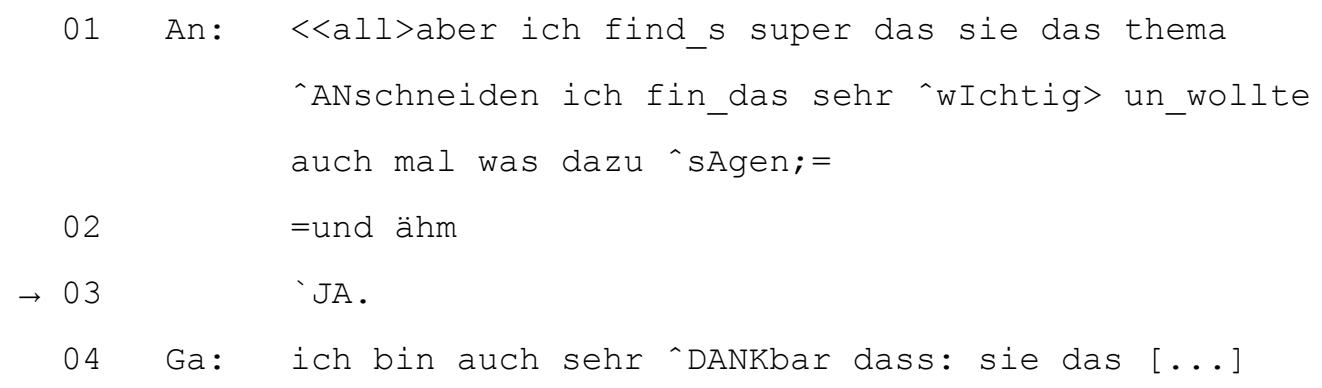

Der Anrufer spricht ein Lob an die Redaktion der Sendung aus, das die Thematisierung der Prostitution befürwortet. Anschließend ist er sich nicht ganz sicher, ob er noch etwas hinzufügen soll (und ähm). Nachdem er die Äußerung abbricht, bestätigt er mit dem ja, dass sein Redebeitrag nach dazu sagen in Zeile 01 eigentlich vollständig war und nach dieser Stelle abgeschlossen werden sollte. Der Gast der Sendung reagiert in Zeile 04 mit einem Lob an den Anrufer. 


\section{$3 \quad$ Stocken beim Wortfinden}

Das Sprechen stellt die komplexeste menschliche kognitiv-motorische Fertigkeitdar (cf. Indeferey/Levelt 2000: 845). Es hat sich evolutionär entwickelt, immer komplexer werdenden sozialen Aktivitäten des Menschen gerecht werdend. Neurophysiologisch lässt sich dessen Steuerung im Wesentlichen in drei Hirnarealen und Interaktionen, die zwischen ihnen ablaufen, lokalisieren: In der Windung der Großhirnrinde im hinteren Teil des unteren linken Parietallappens - dem sog. Gyrus angularis, im mittleren Teil der mittleren Windung des linken Temporallappens - dem sog. Wernicke-Areal und in der Windung der Großhirnrinde im hinteren Teil des unteren linken Frontallappens - dem sog. Broca-Areal. Der Gyrus angularis verarbeitet im Wesentlichen konkrete Stimuli zu abstrakten Konzepten der Außen- und Innenwelt (cf. Seghier 2013: 48). Im Wernicke-Areal werden Konzepte mit entsprechenden Lemmata verknüpft, die in das Broca-Areal weitergeleitet werden, das über phonologische Formen der passenden Wörter verfügt (cf. Indeferey/Levelt 2000: 854, 859). Diese werden in der primären motorischen und der primären sensorischen Hirnrinde zu phonetischen Formen verarbeitet (ibd.: 859).

Das neuronale Korrelat der Selektionsprozesse bei Wortproduktion befindet sich im Wernicke-Areal (ibd.: 854), wo bei eventuellen Wortfindungsproblemen die gewünschten Verknüpfungen nicht oder nicht rechtzeitig erstellt werden.

Es wird hier davon ausgegangen, dass die Sprachproduktion der Schnelligkeit und den lokal wechselnden Anforderungen in Gesprächen untergeordnet ist und dass sie deshalb dynamisch und flexibel verläuft. Dass man spricht, bevor man eine passende komplexe Konstruktion parat hat, beweisen sog. Linksversetzungen (cf. Selting 1993; Scheutz 1997), die lediglich den thematischen Rahmen für eine nachfolgende Äußerung festlegen. Verzögerungen und Pausen vor dem Anschluss mitten in einer angefangenen Äußerung können nur als Spuren der Wortfindungsprobleme interpretiert werden. Sie zeugen außerdem davon, dass man sich häufig erst für eine sprachliche Konstruktion, im Sinne von komplexen und schematischen Konstruktionen in der Konstruktionsgrammatik (cf. Croft 2001: 17), entscheidet, um sie (zumindest zum Teil) erst danach mit Lexik zu versehen. Folgendes Beispiel aus einer Phone-In-Sendung zum Thema Sterbehilfe veranschaulicht meine Annahmen bezüglich der Verzögerungen und Pausen vor dem Anschluss in einer Äußerung:

Transkript 8: „Sterbehilfe“

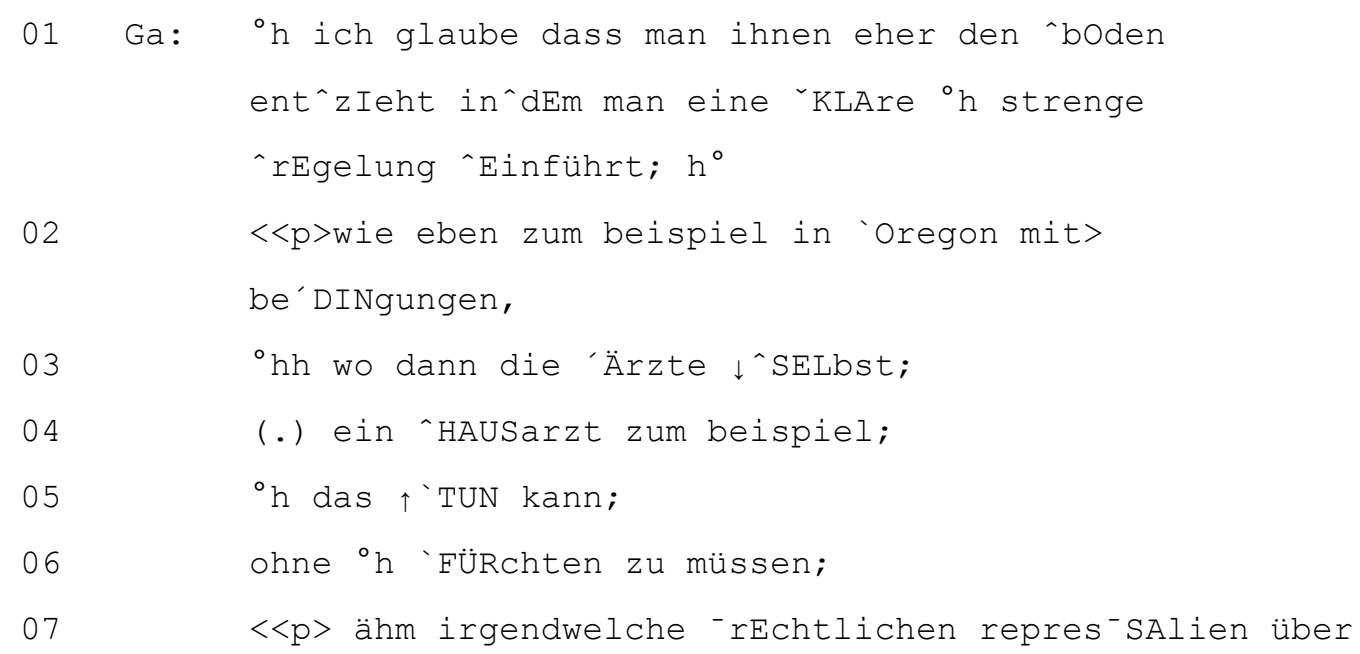




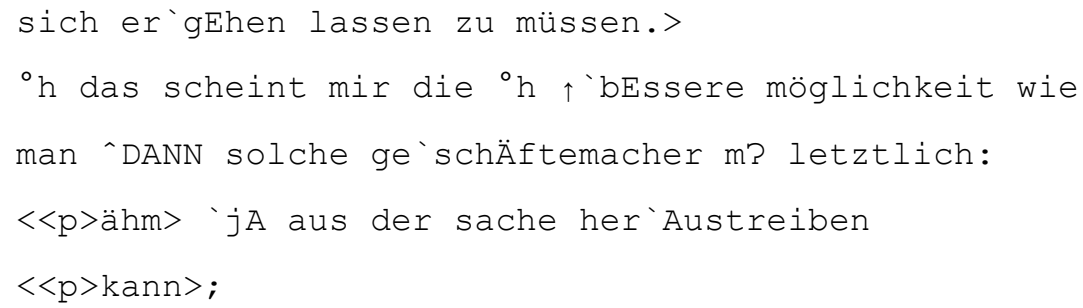

Der Gast der Sendung befürwortet eine gesetzliche Regelung, die nur Ärzte zur Sterbehilfe berechtigen würde, da nur sie kompetent und verantwortungsvoll über die Berechtigung des Unterlassens lebenserhaltender Maßnahmen bei Kranken entscheiden können. Damit würde man die Profitgierigen, die selbst keine Mediziner sind, aus diesem Geschäft los. Abschließend resümiert er (Z. 08) und gibt innerhalb des Attributsatzes zu möglichkeit ein Verzögerungssignal ähm, bevor ihm der Rest der Äußerung, nämlich das Präpositionalobjekt aus der sache und das Prädikat heraustreiben kann, einfällt. Die Generierung des Attributsatzes in Zeile 08 stellt m. E. folgendes Kontinuum zwischen der komplexen, schematischen Konstruktion und der komplexen, spezifischen (und nicht idiosynkratischen) Konstruktion dar:

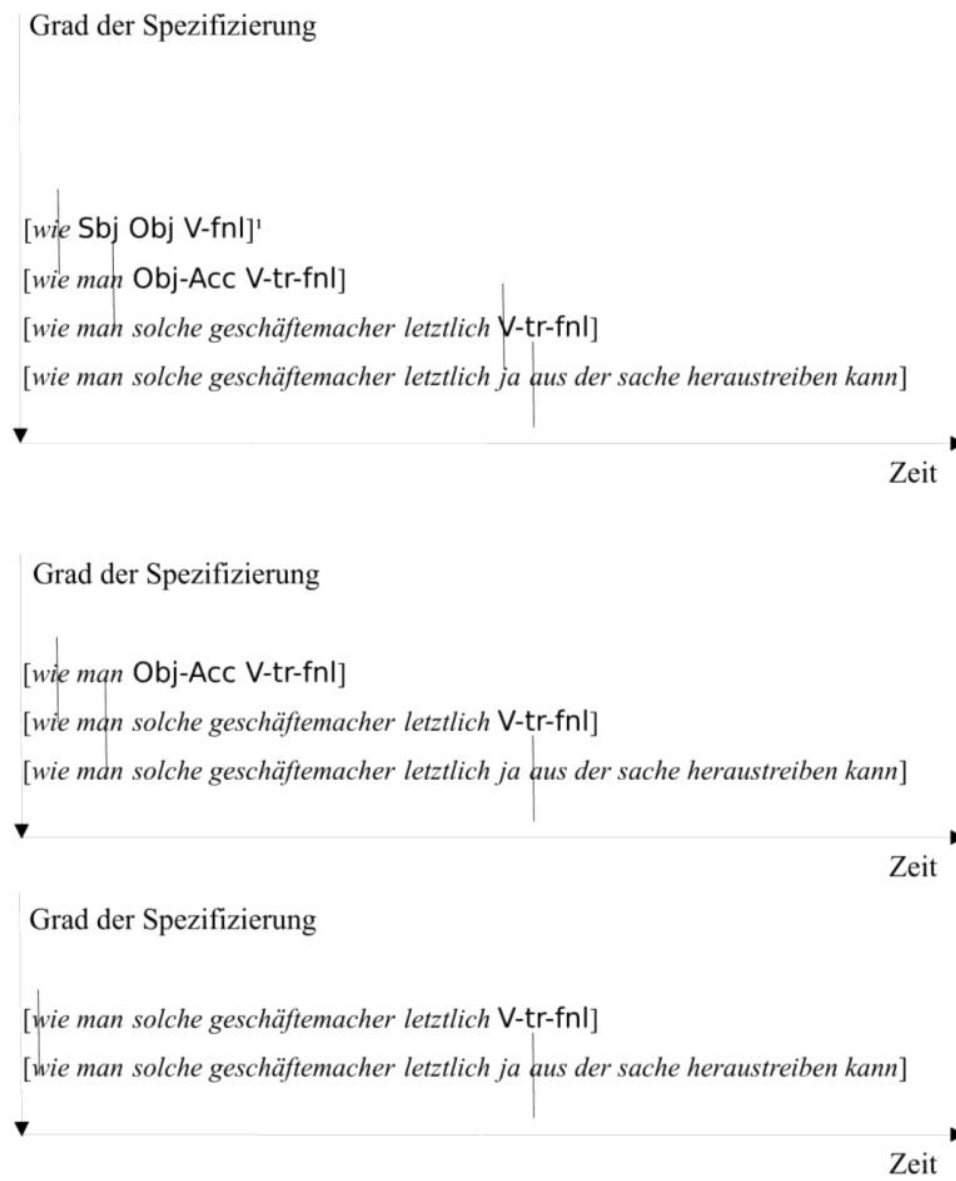

Abbildung. 1. Diagramme mit möglichen Generierungsstadien des Teilsatzes Wie man solche Geschäftemacher letztlich ja aus der Sache heraustreiben kann. Die Senkrechte stellt den Zeitpunkt dar, für den die Konstruktion gilt. ${ }^{1}$

${ }^{1}$ SBJ - Subjekt; OBJ - Objekt; V-FNL - Verb in Letztstellung; OBJ-ACC -Akkusativobjekt ; V-TR-FNL transitives Verb in Letztstellung. 


\section{$4 \quad$ Sprecherwechsel-Regelwerk}

Dass sich die Gesprächsbeteiligten beim Reden meist reibungslos abwechseln, ohne beim Ergreifen des Wortes zu zögern oder nachzufragen, ob der letzte Sprecher seinen Redebeitrag definitiv beendet hat, liegt daran, dass sich die Vergabe des Rederechts voraussagen lässt. Abfallende Stimme (Kadenz) vor dem möglichen Ende einer Äußerung, die im jeweiligen Kontext sinnvoll erscheint und eine Sprechhandlung zum Abschluss bringt, deutet auf eine Vergabe des Rederechts hin. ${ }^{2}$ Eine weiterweisende (relativ gleichbleibende, leicht fallende oder steigende) Stimmführung vor dem möglichen Ende einer Äußerung, die im jeweiligen Kontext selbst sinnvoll erscheint und eine Sprechhandlung zum Abschluss bringen könnte, suggeriert hingegen, dass weitergesprochen wird. Vor ausgeführten Sprechhandlungen und insbesondere vor möglichen Enden der Äußerungen, die eine Sprechhandlung nicht abschließen, ist also das Rederecht ziemlich gesichert. Wenn aber der Redefluss ins Stocken gerät und gerade ein Verzögerungssignal (äh, ähm, Wortwiederholungen, Lautdehnungen usw.) oder eine Pause andauert, wird entweder ein neuer Formulierungsversuch des Sprechers oder Wortfindungshilfen bzw. das Ergreifen des Rederechts von anderen Gesprächsbeteiligten erwartet.

Dass die potentiellen nächsten Sprecher bestenfalls nach möglichen Enden der Äußerungen (bei Abgeschlossensein aller bisherigen Projektionen) legitim das Wort ergreifen können hielten für das Englische erstmals Sacks, Schegloff und Jefferson (1974) fest. Folglich stellen diese möglichen Äußerungsenden für Gesprächsbeteiligte übergabe-relevante Stellen dar (cf. "transition relevance place", ibd.: 703). Sacks, Schegloff und Jefferson erkannten ebenfalls die Rolle der Intonation für das Behalten des Rederechts nach möglichen Äußerungsenden (ibd. 721-722). Dass in ihrem Korpus möglichen Äußerungsenden oft kein Sprecherwechsel folgte sondern den zu Ende ausgeführten Sprechhandlungen, veranlasste Ford und Thompson (1996) zu einer Neudefinition der übergabe-relevanten Stelle. Sie haben sie nämlich um pragmatische Abgeschlossenheit ("pragmatic completion", ibd.: 148-151) erweitert (cf. "complex transition relevance place", ibd.: 154). Folgendes Beispiel aus einer Radio-PhoneIn-Sendung zum Thema Glaubwürdigkeitskrise der Kirchen veranschaulicht alle erwähnten für die Vergabe des Rederechts für relevant gehaltenen Stellen:

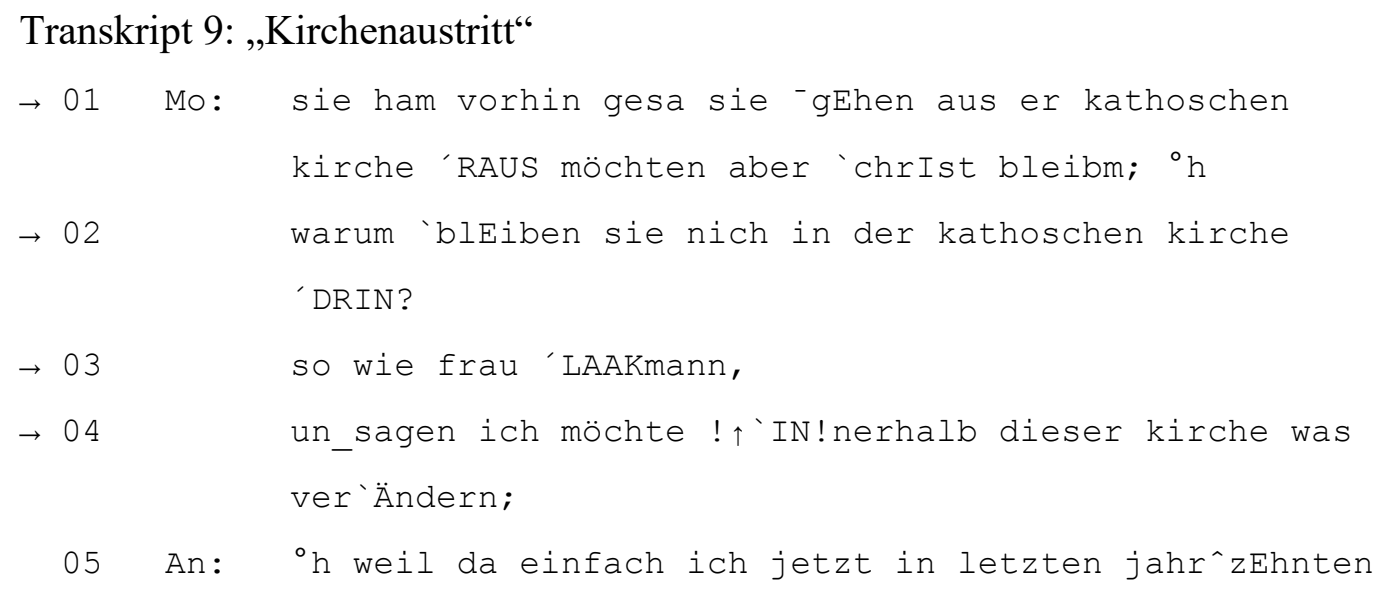

2 Sprechhandlungen, die eine Erwiderungserwartung hervorrufen (konditionelle Relevanz aufbauen) und die in Frageform ausgeführt werden, wodurch eine noch frühere Erwiderung erwartet wird (Marić 2013: 78-79), können im Deutschen intonatorisch u. a. auch steigend und leicht fallend enden (ibd.: 98-100; Selting 1995: 232-307).

ISSN 1615-3014 
06

oder $\uparrow^{`}$ FESTgestellt habe,

${ }^{\circ} \mathrm{h}$ dass Eben $[\ldots]$

Die Moderatorin refokussiert die Aussage des Anrufers aus dem Vorgespräch, dass er aus der katholischen Kirche austreten, trotzdem aber Christ bleiben möchte (Z. 01). Damit bereitet sie auf ihre bevorstehende Sprechhandlung vor, zu deren Präsupposition der Austritt des Anrufers aus der katholischen Kirche gehört. Die Moderatorin schlägt dem Anrufer eine andere Möglichkeit, die eigene Unzufriedenheit mit der katholischen Kirche zu äußern, vor. Ihm wird nämlich geraten, aus der katholischen Kirche nicht auszutreten (Z. 02 und 03) und zu versuchen, innerhalb der Kirche etwas zu verändern (Z. 04). Mögliche Äußerungsenden oder übergaberelevante Stellen ("TRPs - transition relevance places") nach Sacks, Schegloff und Jefferson (1974) gibt es im Gesprächsausschnitt in Zeile 01 nach kirche, nach RAUS, nach bleibm, in Zeile 02 nach kirche und nach DRIN, in Zeile 03 nach LAAKmann und in Zeile 04 nach verÄndern. Vor diesen Stellen verläuft weiterweisend entweder eine ebene, steil ansteigende, leicht ansteigende oder leicht fallende Intonation, niemals aber eine (tief) fallende. Als tatsächliche Äußerungsenden oder Enden der Turnkonstruktionseinheiten ("turn constructional unit - TCU“, Sacks/Schegloff/Jefferson 1974: 701) können retrospektiv die Stellen nach bleibm, nach LAAKmann und nach verÄndern festgestellt werden. Als tatsächliches Redebeitragsende der Moderatorin erweist sich wiederrum die Stelle nach verÄndern, die tatsächlich eine komplexe übergabe-relevante Stelle ("CTRP") darstellt; die Refokussierung der Aussage des Anrufers aus dem Vorgespräch (Z. 01) wird nicht in Form einer Nachfrage realisiert (cf. Rost-Roth 2006: 245-248) und kann lediglich als eine Vorbereitung auf eine bevorstehende Sprechhandlung interpretiert werden; der Teil des Vorschlags aus den Zeilen 02 und 03 ist kein ernstzunehmender, bevor eine Möglichkeit, die eigene Unzufriedenheit mit der katholischen Kirche zu äußern und dadurch zu Änderungen innerhalb der Kirche beizutragen, in Zeile 04 genannt wird.

Das Ergreifen des Rederechts vor einem möglichen Äußerungsende wird im Deutschen von Gesprächsbeteiligten außer bei korrigierenden Entgegnungen als Unterbrechung behandelt (cf. Selting 1995: 208-224). Als solches ist es während eines Verzögerungssignals oder einer Pause, die infolge der Wortfindungsprobleme vor einem möglichen Äußerungsende entstehen kann, nur vereinzelt zu erwarten. Ein Beispiel für eine Unterbrechung entstammt einer RadioPhone-In-Sendung zum Thema selbst erneuerbare Energieerzeugung in Deutschland:

Transkript 10: ,jemand in der Leitung““

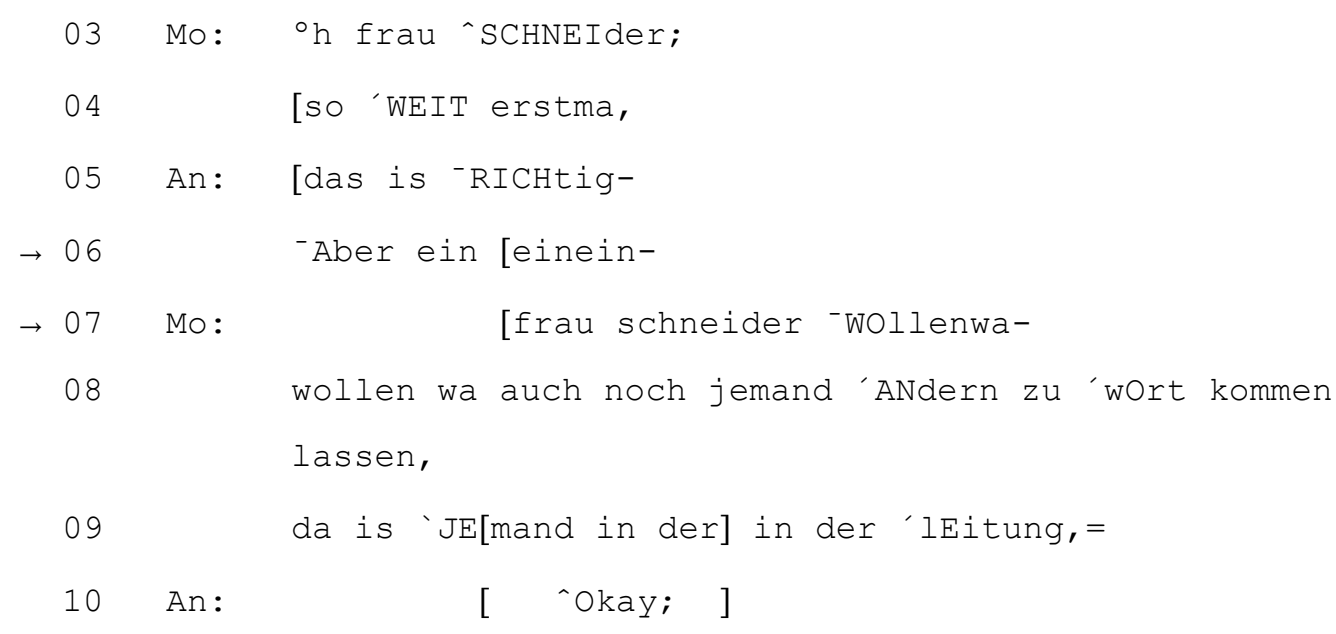

ISSN 1615-3014 
Der Moderator verweigert in Zeile 03 der Anruferin das Rederecht und somit die Möglichkeit zum letzten Redebeitrag des Vorredners Stellung zu nehmen. Er deutet ihr außerdem in Zeile 04 an, dass ihr Beitrag zur Sendung sich seinem Ende nähert. Diese Andeutung überlappt mit ihrer partiellen Zustimmung mit dem Vorredner, durch welche sie ihren Widerspruch einleitet. Sie schafft es ihren Widerspruch gerade anzufangen, bevor sie vom Moderator bei der Wortsuche (ein ein ein) unterbrochen wird (Z. 06). Diese Unterbrechung zielt darauf ab, das Wort zu ergreifen, um das Gespräch mit der Anruferin schneller zu beenden. In seinem Redebeitrag fordert er sie nämlich dazu auf, die weiteren Rederechtansprüche zu unterlassen (Z. 07 und 08), und begründet anschließend seine Aufforderung durch eine/n wartende/n Anrufer/in in der Leitung (Z. 09). Die Anruferin akzeptiert den Verlust des Rederechts durch Unterbrechung in Zeile 06 sowie das Untersagen des weiteren Rederechts zwecks Gesprächsbeendigung in Zeile 10.

Nach möglichen Äußerungsenden ("possible completion onset", Jefferson, 1986: 154-156) oder ganz kurz davor ("last-item onset", ibd.: 157-158) sind zumindest im Englischen Versuche der Rederechtübernahme, die sich mit einem andauerndem Redebeitrag überschneiden, relativ häufig. Dies berechtigt zu glauben, dass im Deutschen während einer Pause, die bei Wortfindungsproblemen unmittelbar nach möglichen Äußerungsenden entstehen kann, mit Versuchen der Rederechtübernahme zu rechnen ist. Der folgende Ausschnitt aus einem Radiogespräch über die Medienaffäre des damaligen Bundespräsidenten Wulff veranschaulicht eine Überlappung erzeugende Rederechtübernahme nach möglichem Äußerungsende:

Transkript 11: „Tabu“

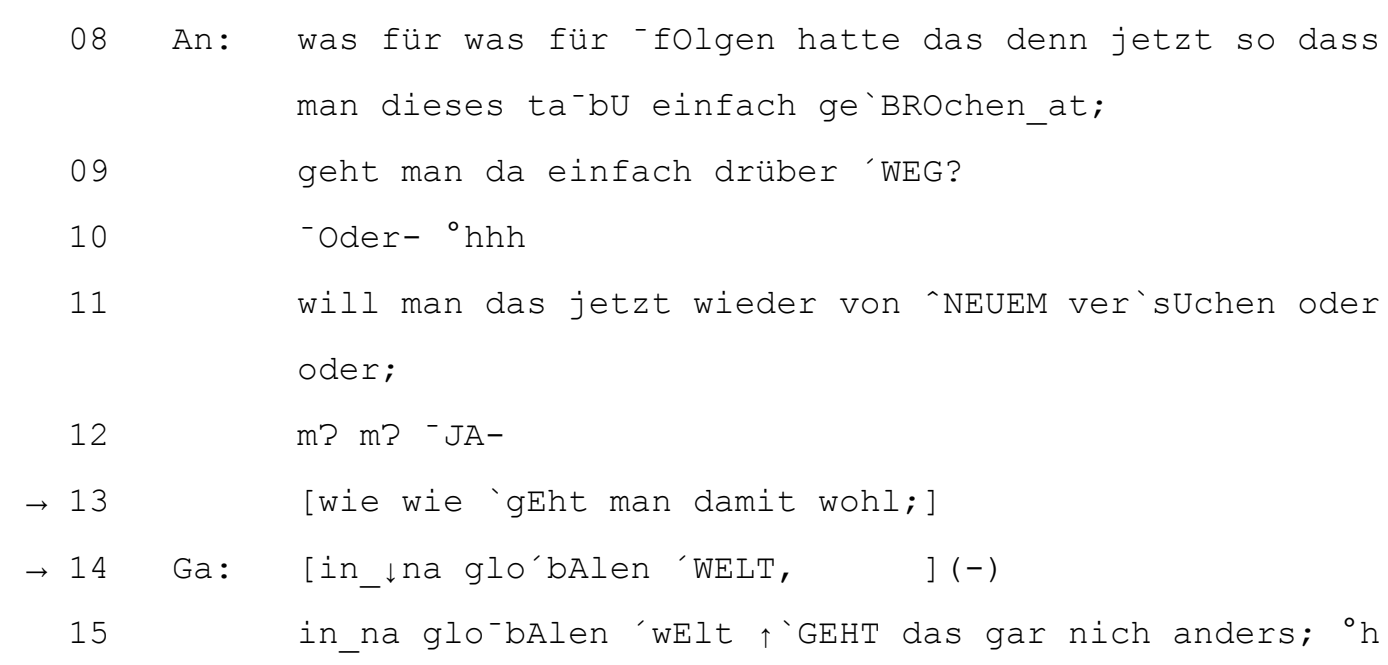

Der Anrufer wirft den im Studio anwesenden Medienvertretern vor, das davor erwähnte Abkommenunter Medien (die Politiker nicht anzugreifen, wenn sie auf Auslandsreisen sind) gebrochen zu haben. Er wirft ihnen außerdem vor, gegen das Tabu, jemanden zu kritisieren, der nicht da ist und sich nicht wehren kann, verstoßen zu haben. Er möchte wissen, wie damit im Nachinein umgegangen wird (Z. 08, 09 und 13) sowie, wie in Zukunft in ähnlichen Fällen vorgegangen wird (Z. 10 und 11). Die Alternativfrage in Zeilen 09, 10 und 11 fungiert als eine Vorwegnahme der Antwort auf seine Frage aus der Zeile 08, da es nach zwei komplexen übergabe-relevanten Stellen (nämlich nach Auslandsreisen sind und nach geBROchen_at) nicht zum gewünschten Sprecherwechsel kommt. Dadurch werden zwei neue komplexe übergabe-relevante Stellen geschaffen: nach WEG? und nach versUchen. Erst nach dem 
Verzögerungssignal oder oder und dem Aufforderungssignal - $J A$ - fängt der Gast der Sendung zeitgleich mit einem erneuten Versuch des Anrufers, das Rederecht zu vergeben, mit dem Zurückweisen des Vorwurfs an. Nach anfänglicher Überlappung wird ihm, wie erwartet, das Rederecht eingeräumt. Das Zögern mit dem Zurückweisen des Vorwurfs ist der Tatsache zuzuschreiben, dass im Studio mehrere Medienvertreter als nächste Sprecher in Frage kamen.

Pausen, die vor dem Anschluss-ja vorkommen, können eigentlich bei genauem Hinhören und Hinsehen als turninterne erkannt werden, weil sie im Gegensatz zu postfinalen, die ein hörbares Ausatmen ausmacht, infolge gehaltenen Glottalverschlusses entstehen (cf. Local/Kelly 1986: 195). Selting (1995: 63) kommt diesbezüglich für das Deutsche zum gleichen Ergebnis. Diesen Pausen gehen außerdem Konstruktionskonstituenten voraus, die ein gleichbleibender Tonhöhenverlauf kennzeichnet, eventuell auch Lautdehnungen und Verzögerungssignale wie äh, öhm (ibd.: 63).

Nach einem Verzögerungssignal und einer Pause, die einer abgebrochenen Äußerung folgt, kann ebenfalls eine Selbstkorrektur in Form eines Neuanfangs erwartet werden, weil diese in dieser Kombination eben häufig vorkommen (ibd.: 68). Möglich ist natürlich auch, dass Verzögerungssignale ohne Pause danach oder eine Pause ohne Verzögerungssignal davor im Anschluss an eine abgebrochene Äußerung entstehen. Das Beispiel für einen Neustart nach einem Satzbruch und Verzögerungssignalen entstammt einem Radiogespräch über eine Lohnuntergrenze in Deutschland:

Transkript 12: „Gehälter der oberen Einkommensbezieher“

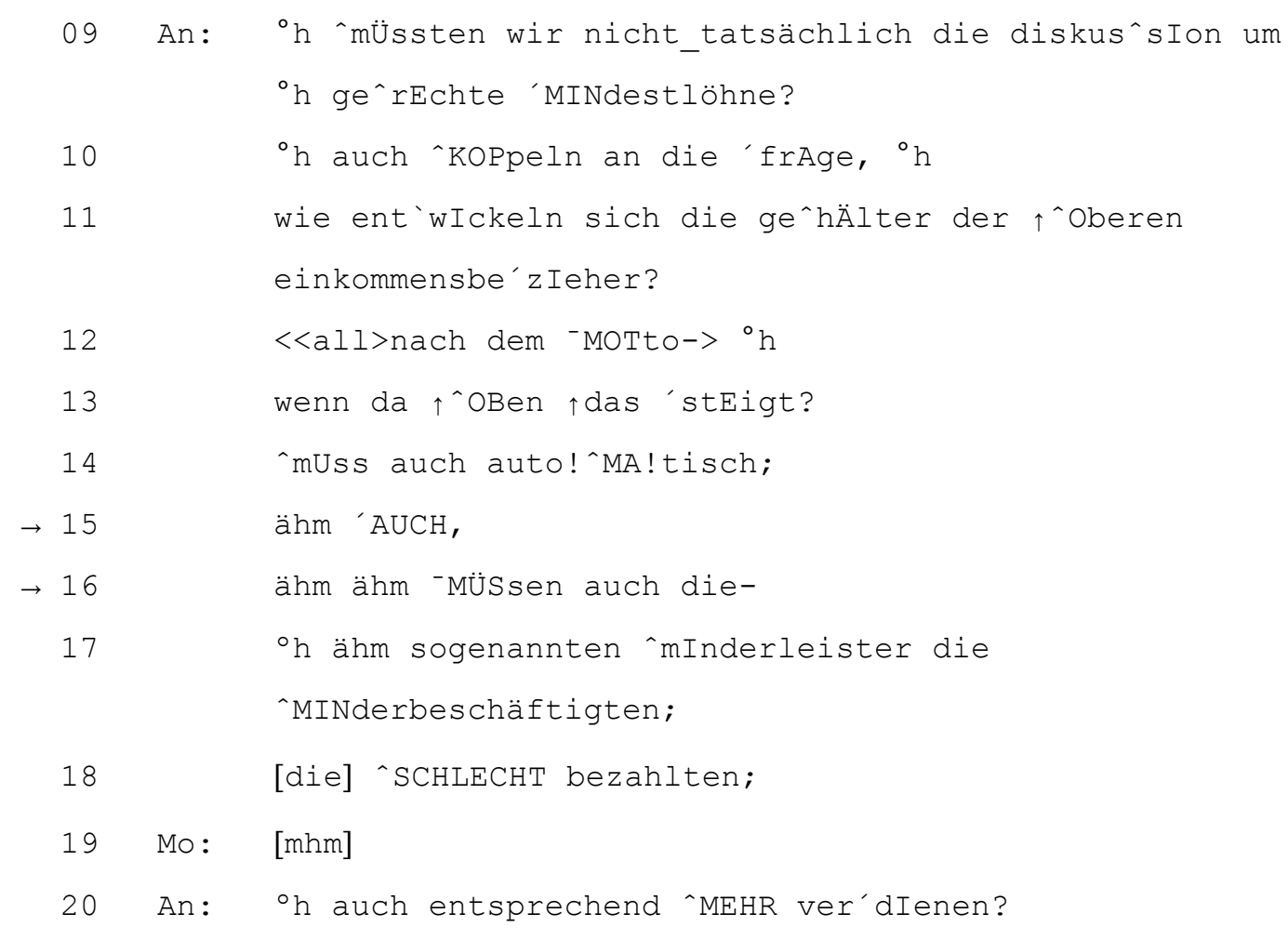

Der Anrufer macht einen Vorschlag für eine mögliche Lösung des Problems der zunehmenden Gruppierung um die Pole Reich und Arm innerhalb der deutschen Bevölkerung. Die Lösung setzt einen proportionalen Anstieg der Mindestlöhne und der Löhne der Gutverdiener voraus.Der neue Aspekt der Bindung zweier Lohngruppen (Z. 10 und 11) wird in der Abfolge eines Konditionalsatzes (Z. 13) und des dazugehörigen Hauptsatzes (Z. 
14) näher erläutert. Da der Anrufer kein Subjekt für die angefangene Konstruktion müssen + Modalangabe +Subjekt +Vollverb parat hat, bricht er sie ab (Z. 16). Dabei hinterlässt er Verzögerungssignale ähm AUCH ähm ähm, um anschließend die Form der 3. Person Singular des Verbs müssen durch die Form der 3. Person Plural desselben Verbs in einer neuen Äußerung (Z. 16) zu ersetzen. Dass sich der Anrufer in Zeile 16 nur des grammatischen Numerus des Subjekts sicher ist, zeigen Verzögerungssignale in Zeilen 16 und 17 auch, ${ }^{\circ}$, ähm und sogenannten sowie eine sich fortsetzende Wortsuche: mInderleister die MINderbeschäftigten die SCHLECHT bezahlten. Der Abbruch und der Neustart sind hier also aufgrund der Wortfindeprobleme entstanden und nicht aufgrund der beabsichtigten Selbstkorrektur der Verbform.

Wortfindungshilfen von anderen Gesprächsbeteiligten sind auch keine Seltenheit nach Pausen und Verzögerungssignalen, die unterbrochenen Äußerungen folgen. Im folgenden Gesprächsausschnitt aus einer Radio-Phone-In-Sendung kontextualisiert die Moderatorin durch leises Sprechen ihre Wortfindungshilfe als einen Beitrag ohne Turnbeanspruchung (Selting 1995: 224-229):

Transkript 13: „Statistiken“

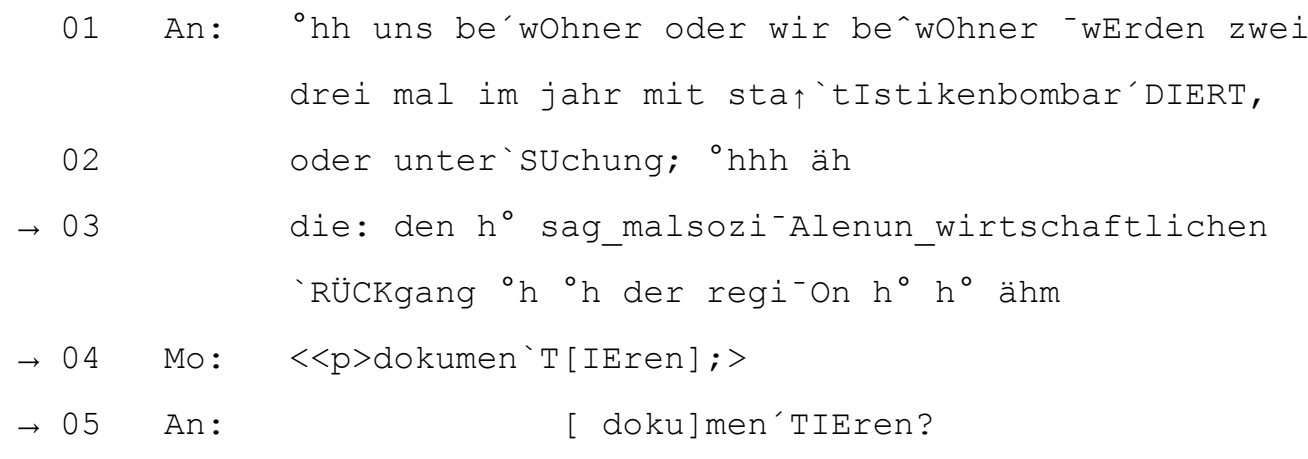

Wortfindeprobleme lassen sich in Zeile 03 an häufigem Ein- und Ausatmen um die unbesetzte Verbstelle der Konstruktion und am Verzögerungssignal ähm erkennen. Der Anrufer akzeptiert sofort die Wortsuggestion der Moderatorin, indem er das Wort in seine unterbrochene Äußerung intonatorisch integriert (Z. 05).

\section{$5 \quad J a$ als Turnhalte- und Anschlusssignal}

\subsection{Definitionen des Anschluss-ja und Analysedaten}

Das turnintern eingesetzte $j a$, dem Spuren der Wortfindungsprobleme wie z. B. Verzögerungssignale (äh, ähm, Wortwiederholungen, Lautdehnungen usw.), Pausen oder hörbare Atemzüge vorausgehen, wurde bei der Datenerhebung der Kategorie der Anschluss-ja zugeordnet. Es wird hier davon ausgegangen, dass es bei Wortfindungsproblemen zur Ankündigung einer Vervollständigung einer vorausgehenden Äußerung und gleichzeitig zur Beibehaltung der Sprecherrolle eingesetzt wird.

Das Korpus dieser Untersuchung stellen 100 das Anschluss-ja jeweils enthaltende Ausschnitte aus den Gesprächen. Diese wurden in der Phone-In-Sendung „Im Gespräch“ des Deutschlandradios zwischen den Moderatoren, den Gästen und den Anrufern meist in den jahren 2014 und 2015 geführt. Für die Notation der Daten wurde die Notationskonvention des 
GATs 2 (cf. Selting et al. 2009) verwendet. Mithilfe einer qualitativen Sequenzanalyse der Gesprächsausschnitte werden die konversationellen Funktionen des Anschluss-ja genau festgelegt.

\subsection{Analyseergebnisse}

Die Analyse ergab, dass sich die phonetischen Realisierungsvarianten des Anschluss-ja hauptsächlich auf der suprasegmentalen Ebene unterscheiden. Unterschiede bestehen nämlich in der Richtung der Tonhöhenänderung, im Ausmaß der Tonhöhenänderung (ihrer Prominenz), im Tonhöhenregister, in der Stimmfarbe und in ihrem intonatorischen Angepasstsein an das unmittelbar davor und das unmittelbar danach Geäußerte. Auf der segmentalen Ebene betreffen die Variationen eine kurze Realisierung ([a]) des /a:/ in /ja:/ sowie das unmittelbar an die Lautfolge angeschlossene hörbare Ausatmen: jahh ${ }^{\circ}$. Eine für ein fließendes Sprechen unübliche Dehnung des /a:/, also ja:, ja:: oder ja:: kommt im Korpus so gut wie gar nicht vor. Es können ebenfalls weder auditiv noch in Spektrogrammen Geräuschanteile in der Realisierung des / $\mathrm{j} /$ festgestellt werden, die im Wesentlichen das [j] und das [ç] ausmachen. Dafür zeigen Spektrogramme stets die für das [j] charakteristische Verlagerung der Formantenfrequenzen sowie eine geringere Schallintensität des [j], die am weniger intensiven Schwarz der Formanten erkennbar ist (siehe Abbildungen 2 und 3).

Die Formantenwerte der Laute [a:] und [a] in der Phonemfolge/ja:/ befinden sich sowohl bei männlichen als auch bei weiblichen Sprechern jeweils innerhalb der Werterahmen für Formanten des deutschen Lautes [a:] bzw. [a] (cf. Abbildung 3 und Formantkarten des deutschen Vokalsystems von Sendlmeier/Seebode).

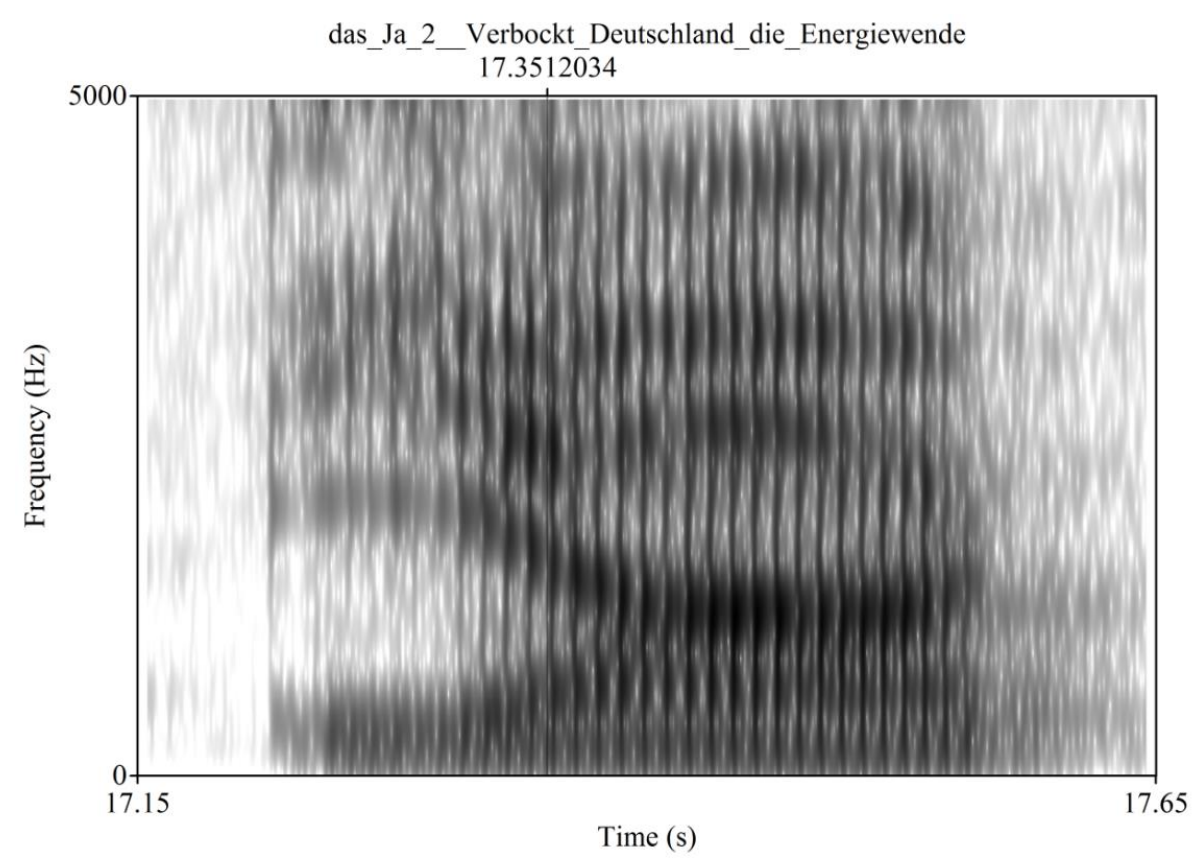

Abbildung 2: Darstellung der Lautfolge [ja:] aus der Äußerung weil's in der Tat äh ja keine ruinösen Kosten sind im Spektrogramm 


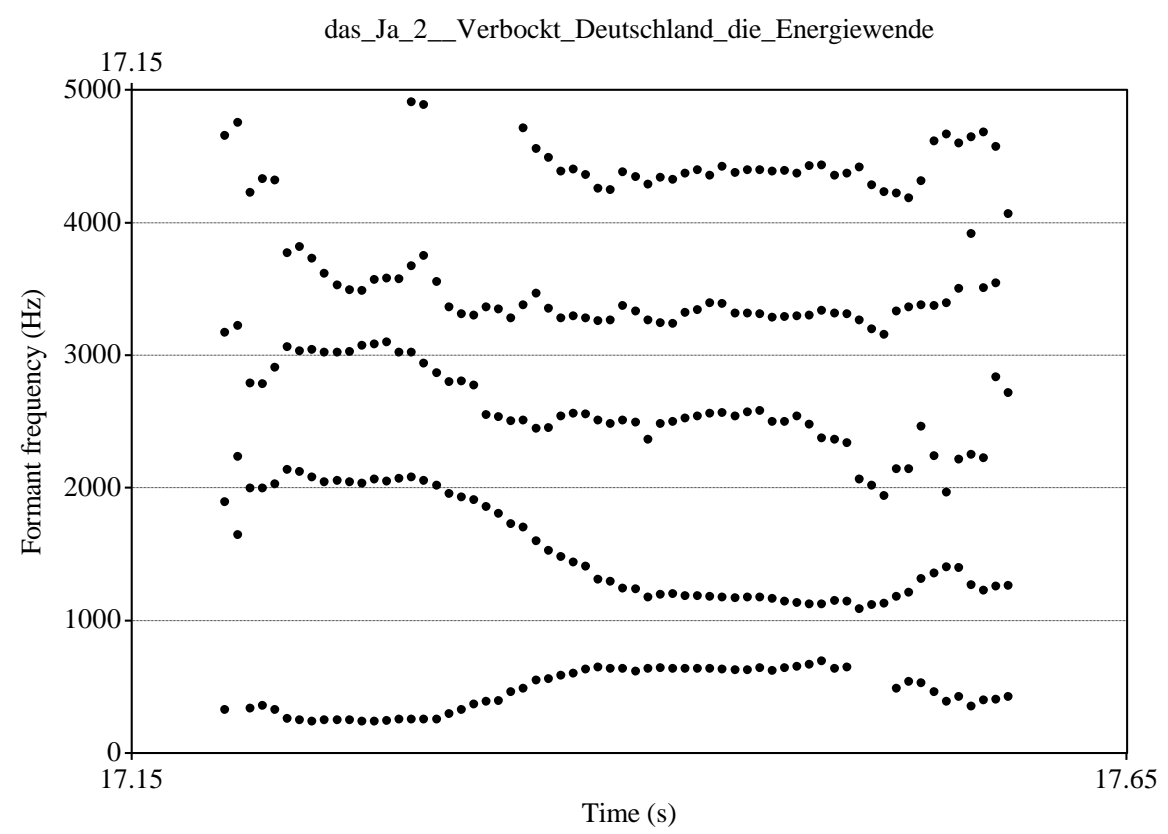

Abbildung 3: Darstellung der Formantenwerte der Lautfolge [ja:] aus der Äußerung weil's in der Tat äh ja keine ruinösen Kosten sind

Die häufigste Erscheinungsform des Anschluss-ja ist eindeutig die auf einer gleichbleibenden oder leicht fallenden globalen Verlaufslinie der Intonation ohne deren hörbare Richtungsänderung (48 Beispiele). Dieser folgen die mit einer hörbaren fallenden Richtungsänderung des Tonhöhenverlaufs, die mehr oder weniger prominent sein kann (24 Beispiele). Bei 17 Anschluss-ja kommt eine mehr oder weniger prominente steigend-fallende Richtungsänderung des Tonhöhenverlaufs vor. Die restlichen 11 Anschluss-ja weisen entweder eine steigende oder fallend-steigende Richtungsänderung des Tonhöhenverlaufs oder einen ebenen lokalen Tonhöhenverlauf mit gleichzeitiger Änderung des Tonhöhenregisters bzw. Änderung der Stimmfarbe auf (kurzer Wechsel zur Knarrstimme, gehauchten Stimme oder nasalen Stimme).

Ein weiteres Unterscheidungsmerkmal, nämlich das intonatorische Angepasstsein des Anschluss-ja an das unmittelbar davor und das unmittelbar danach Geäußerte, erwies sich als

besonders signifikant. Der Tonhöhenansatz des Anschluss-ja, der nicht einmal annäherend der Endtonhöhe der vorausgehenden Äußerung, sowie die Endtonhöhe des Anschluss-ja, die nicht einmal annäherend dem Tonhöhenansatz der Fortsetzung der Rede angeglichen wird (siehe ein Beispiel dazu in Abb. 4.), gehen, wie die Analyse zeigt, mit einer nachfolgenden neuen Satzkonstruktion einher. Diese neue Satzkonstruktion kann sowohl ein Anakoluth als auch einfach eine neue Äußerung nach abgeschlossener Vorläuferäußerung sein. Die Analyse zeigt also, dass ein geäußertes Anschluss-ja nicht immer auf eine nicht erstellte Verknüpfung im Wernicke-Areal oder auf eine nicht zufriedenstellende Verknüpfung im Wernicke-Areal hinweist (siehe Kapitel 3). Sie zeigt ebenfalls, dass Satzabbrüche doch vor den Anschluss-ja vorkommen. Demnach bedeutet das Anschluss-ja nicht unbedingt ,gleich geht's weiter mit der angefangenen Äußerung“ sondern ,gleich geht's weiter“. Der folgende Ausschnitt aus einem Gespräch zum Thema Wulff-Affäre veranschaulicht eine solche Verwendung des Anschluss-ja:

Transkript 14: „Hase und Igel““

10 Ga: und ich 'dEnke mir (---) ((schmatzt)) dass äh ${ }^{\circ}$ hh 


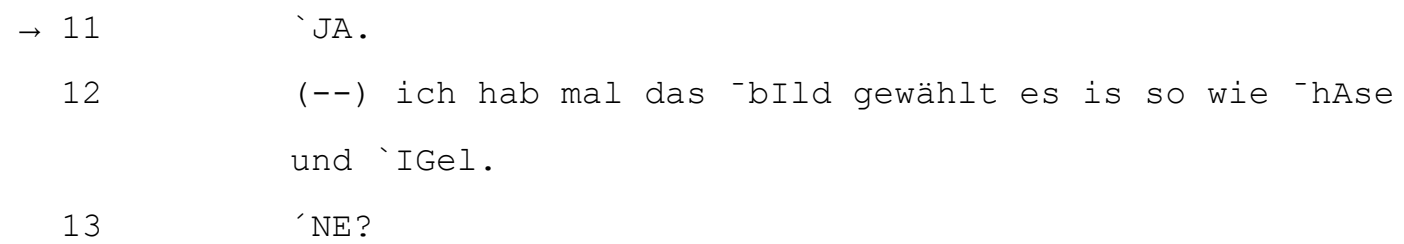

Der Politikwissenschaftler im Studio hat direkt vor diesem Gesprächsausschnitt eine Sequenz abgeschlossen, die die Ursachen für die Wulff-Affäre im Benehmen des damaligen Bundespräsidenten Wulff gegenüber den Medien findet. Dass der Sprecher gleich im Anschluss an diese Sequenz in Zeile 10 keine passende sprachliche Konstruktion parat hat, kann retrospektiv (in Zeile 11) aus dem Satzabbruch geschlossen werden. Nach den Verzögerungssignalen, dem Anschluss-ja und einer Pause kann schließlich die Konstruktion realisiert werden, die die Dementierungsversuche des damaligen Bundespräsidenten Wulff mit dem Handeln des Hasen im Märchen „Hase und Igel“ vergleicht.

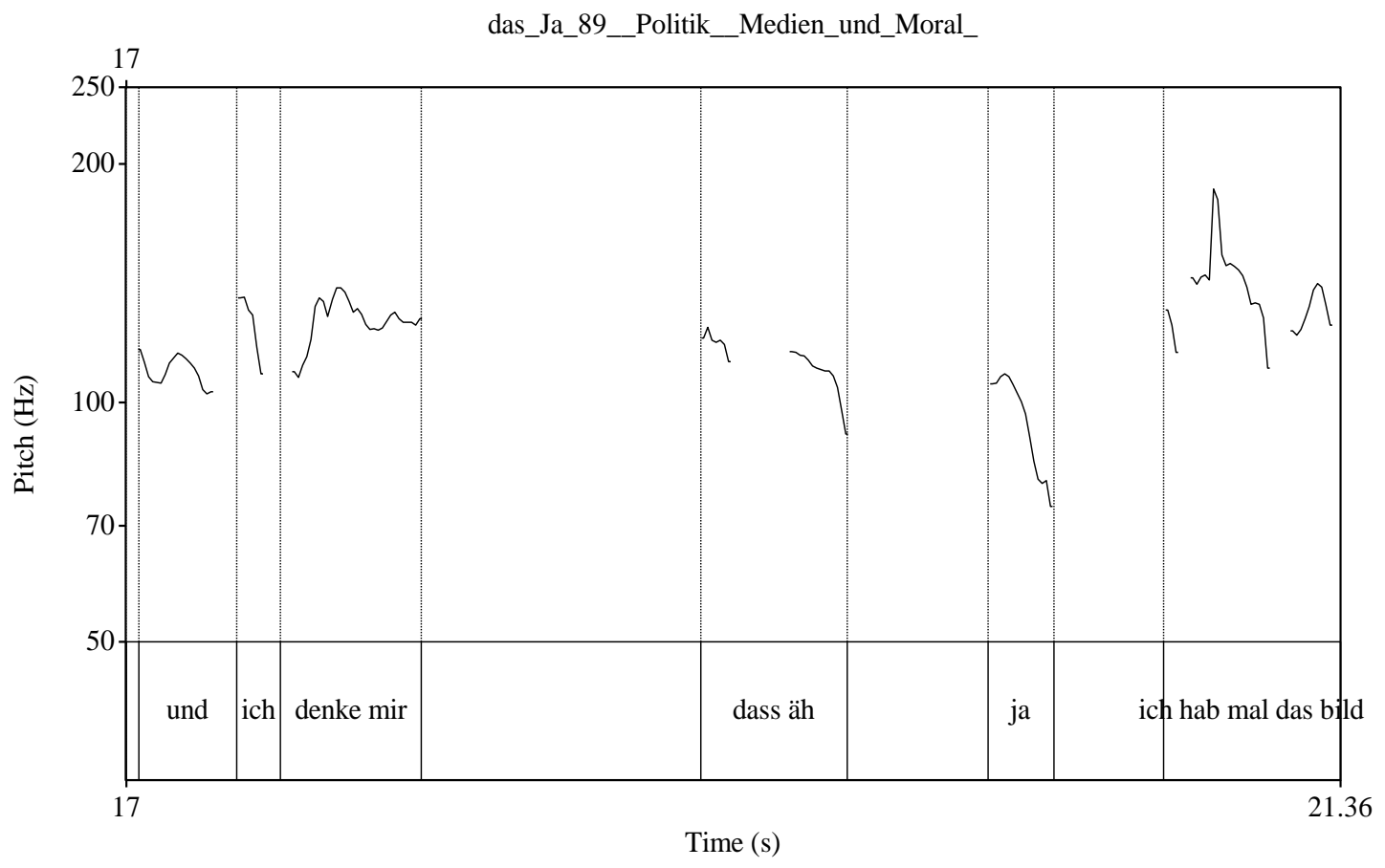

Abbildung 4: Diagramm des Intonationsverlaufs der abgebrochenen Äußerung und ich denke mir dass, des nicht angepassten Intonationsverlaufs von $j a$ und des Teilverlaufs des Neuanfangs ich hab mal das Bildgewählt es ist so wie Hase und Igel.

Der nächste Ausschnitt aus einem Gespräch über Musikalität jedes Einzelnen und musikalische Bildung veranschaulicht die Verwendung des Anschluss-ja nach einer vollständigen Äußerung innerhalb des Redebeitrags:

Transkript 15: „Klettern in den Alpen“

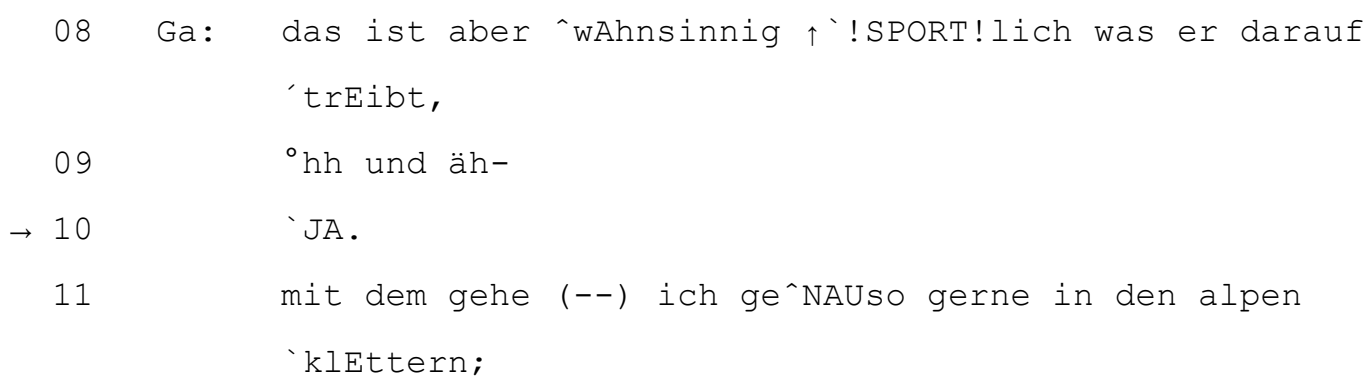


Der Gast im Studio vermittelt in Zeile 08 ein lebendiges Bild von der Art und Weise des Spielens seines Cellisten und Kontrabassisten im Orchester. Anschließend kündigt er durch eine leicht steigende letzte Tonhöhenbewegung, die Konjunktion und (Z. 09) und das Anschluss-ja (Z. 10) eine Fortsetzung an, um schließlich den Aspekt ihrer Freundschaft zu erwähnen, dass sie auch außerhalb des Orchesters gerne ihre Zeit miteinander verbringen.

Vereinzelt folgen intonatorisch unangepasste Anschluss-ja mit möglichen Äußerungsenden, woran Expansionen angeknüpft werden. Aus demselben Gespräch wie der letzte Transkriptausschnitt stammt folgende argumentative Sequenz:

Transkript 16: „die Emotion und die Kreativität““

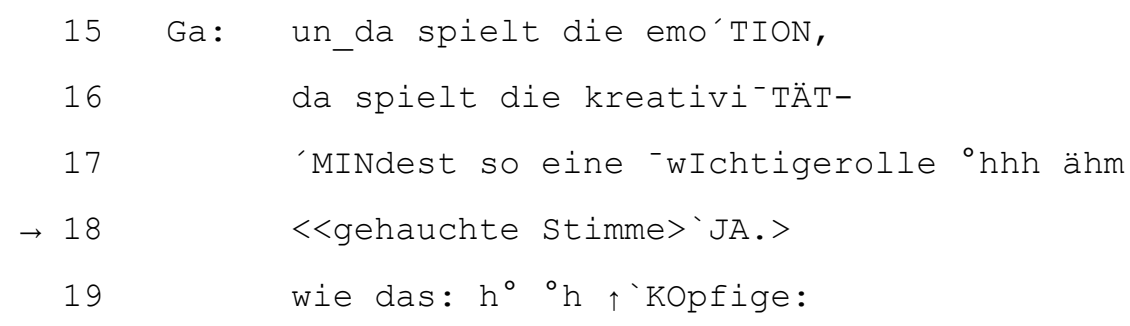

Der Gast im Studio befürwortet die Förderung anderer Fähigkeiten als nur der kognitiven sowie eines anderen Wissens als nur des Faktenwissens in der Bildung. Er zählt die emotionale Intelligenz und Kreativität als der Ratio gleichwertige Fähigkeiten auf. Nach möglichem Äußerungsende nach rolle und einem Verzögerungssignal wird ein Anschluss-ja in der eigenen Intonationsphrase geäußert, woran wie das kopfige angeschlossen wird.

Eigentlich beugt nur das durchaus häufigere (77 Beispiele), intonatorisch sowohl an die unterbrochene Äußerung als auch an die Fortsetzung der Äußerung angepasste Anschluss-ja (siehe ein Beispiel dazu in Abb. 5.) einem vorzeitigen Schließen auf Satzbruch durch den Hörer vor und nicht, wie in der Einleitung angenommen, jedes Anschluss-ja. 


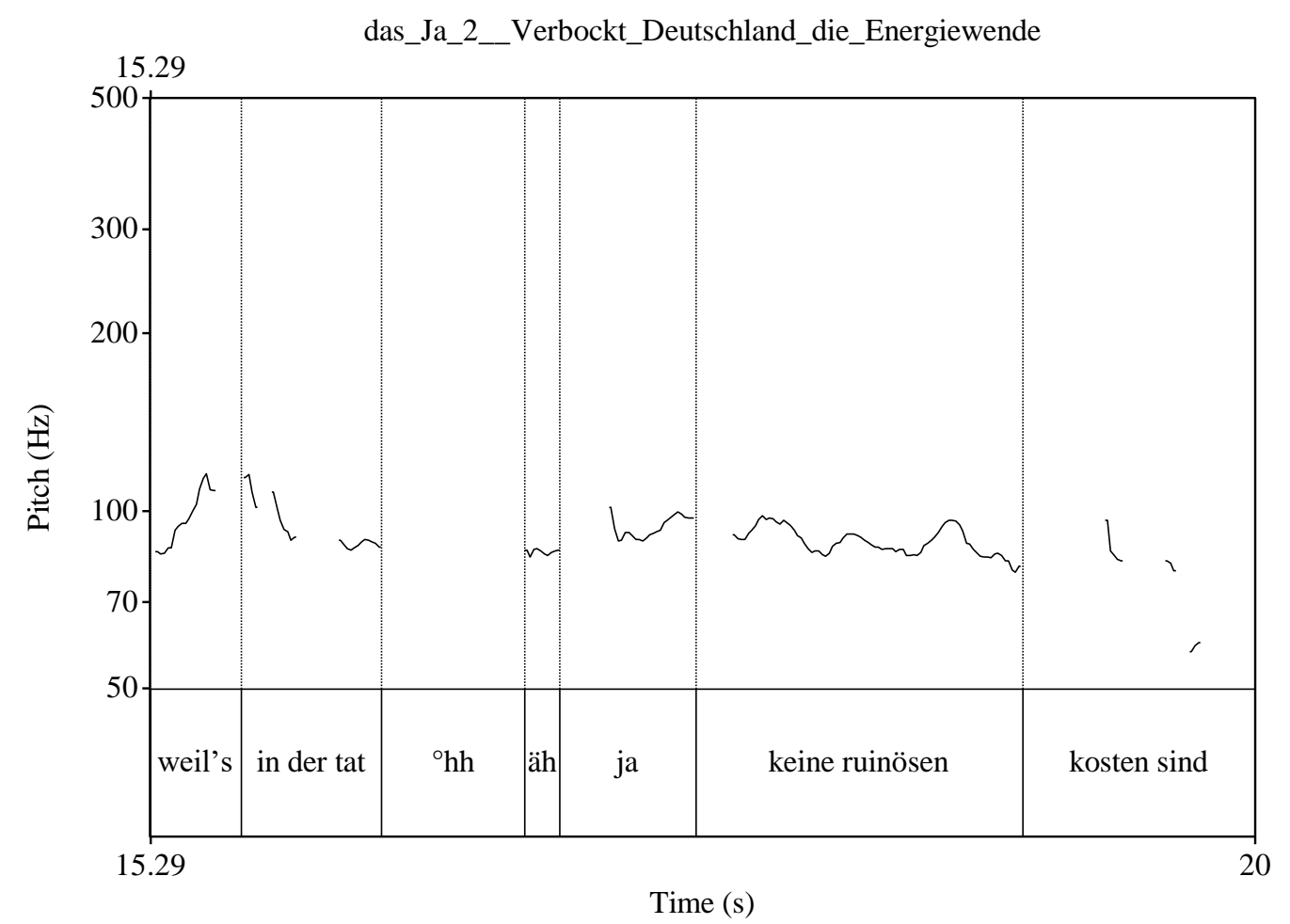

Abbildung 5: Diagramm des Intonationsverlaufs der Intonationsphrase weil's in der Tat äh ja keine ruinösen Kosten sind mit einem Anschluss-ja, das seiner sprachlichen Umgebung intonatorisch angepasst ist.

Außer einer oder mehrerer fehlenden Äußerungskonstituenten, die in der Regel für die Sinnhaftigkeit der Äußerung im jeweiligen Kontext notwendig sind, kündigt das intonatorisch eingefügte Anschluss-ja auch Selbstkorrekturen an. Diese stellen keine völlig neuen Konstruktionen, sondern Korrekturen der einzelnen spezifischen Konstituenten und eventuell den ihnen folgenden Rest der Konstruktion dar. Ebenfalls aus dem Gespräch über Musikalität jedes Einzelnen und musikalische Bildung stammt der folgende Ausschnitt aus einer Befürworten-Sequenz. Sie erklärt das Interesse für Musik jedes einzelnen musizierenden Menschen für die wichtigste Form der Unterstützung ihrer Musik.

Transkript 17: „größte Unterstützung““

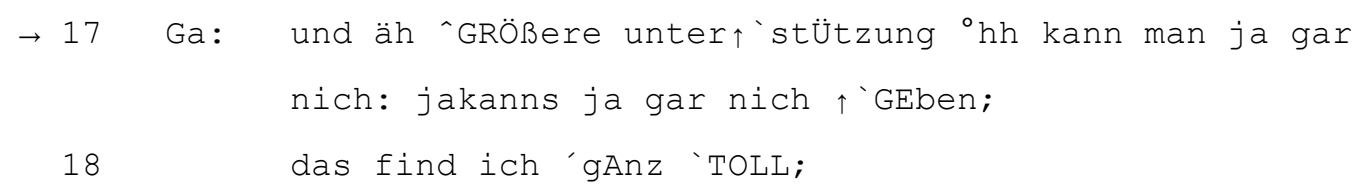

Der Gast im Studio bricht in Zeile 17 den Satz ab und nach einem Anschluss-ja setzt er in die gleiche Konstruktion (größereUnterstützung + kann + SBJ + ja + gar nicht + V-FNL) ein es statt man ein und in die Vollverbstelle das Verb geben, ohne dabei das Akkusativobjekt größere Unterstützung zu wiederholen.

Insgesamt kommt es nur einmal im Korpus vor, dass der Sprecher mit dem nach dem Anschluss-ja geäußerten Wort hörbar unzufrieden ist (Z. 10 quatsch). In einem Gespräch über Lieblingsfilme begründet er sein davor ausgesprochenes Lob an den Filmkritiker, der als Gast an der Sendung teilnimmt. Das passende Verb, das er annimmt (Z. 13), bietet ihm die Moderatorin in Zeile 11 an. 
Transkript 18: „Filmkritiker“

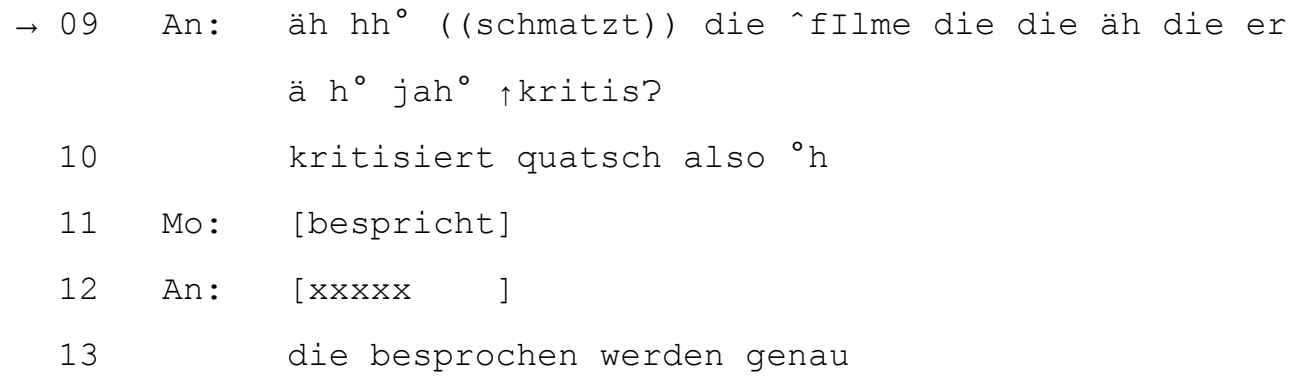

Verzögerungssignale kommen in 18 Beispielen auch nach einem geäußerten Anschluss-ja vor, woraus geschlossen werden kann, dass das Anschluss-ja nicht unbedingt ,jetzt hab' ich's“ bedeutet. Unter diesen 18 Anschluss-ja findet sich so gut wie keines mit einer steigendfallenden oder mit einer fallenden Tonhöhenbewegung mit einem Tonhöhensprung nach oben davor. Die Anschluss-ja mit gerade diesen Tonhöhenverläufen könnten demnach einen Worteinfall, den man ,in der Tasche hat", signalisieren.

Äußerungskonstituenten, die dem Anschluss-ja und eventuellen Verzögerungssignalen folgen und auf diese Weise die Äußerung vervollständigen, reichen von ganzen Teilsätzen über mehrere Satzglieder bis zu Gliedteilen. Die Mehrzahl stellen jedoch einzelne Satzglieder dar und zwar am häufigsten Prädikate bzw. Prädikatsteile, Objekte und Prädikative, etwas seltener Subjekte und vereinzelt Lokal-, Modal-, und Kausalbestimmungen, Vergleichsergänzungen und Attribute.

Abschließend bleibt noch zu erwähnen, dass Anschluss-ja in der Regel in längeren Redebeiträgen wie argumentativen Sequenzen, Erzähl-, Schilderungs-, Interpretations-, Lobes-, Kritik-, Begründungs-, Widerspruchs-, Befürwortungs-, Erkundigungs-, Auskunftund Vorschlags-Sequenzen vorkommen. Das Rederecht des Sprechers während des Stockens und unmittelbar danach war außerdem niemals durch Versuche der Rederechtübernahme der Gesprächsbeteiligten gefährdet.

\begin{tabular}{|l|l|l|}
\hline $\begin{array}{l}\text { intonatorisches } \\
\text { Angepasstsein }\end{array}$ & angekündigte Folgeeinheit & Anzahl \\
\hline $\begin{array}{l}\text { intonatorisch unangepasste } \\
\text { Anschluss-ja }\end{array}$ & $\begin{array}{l}\text { Neuanfang nach einem } \\
\text { Satzabbruch }\end{array}$ & 16 \\
\cline { 2 - 3 } & neue Satzkonstruktion & 6 \\
\cline { 2 - 3 } & $\begin{array}{l}\text { Expansion der } \\
\text { Vorgängeräußerung }\end{array}$ & 1 \\
\hline $\begin{array}{l}\text { intonatorisch angepasste } \\
\text { Anschluss-ja }\end{array}$ & $\begin{array}{l}\text { fehlende } \\
\text { Äußerungskonstituente }\end{array}$ & 73 \\
\cline { 2 - 3 } & $\begin{array}{l}\text { Selbstkorrektur einer } \\
\text { Konstituente }\end{array}$ & 4 \\
\hline
\end{tabular}

Tabelle 1: Tabellarische Darstellung des Zusammenhangs zwischen dem intonatorischen Angepasstsein des Anschluss-ja an seine sprachliche Umgebung und der Art der angekündigten Folgeeinheit mit der Anzahl der Belege im Korpus.

\section{$6 \quad$ Fazit}

Die Datenanalyse hat ergeben, dass das Anschluss-ja allgemein ,gleich geht's weiter" bedeutet und in der Regel in längeren Redebeiträgen, die sowohl kooperative als auch antagonistische Sprechhandlungen ausmachen können, vorkommt. 
Die phonetische Realisierungsvariabilität des Anschluss-ja betrifft hauptsächlich seine suprasegmentale Ebene: Änderungsrichtung(en) seines Intonationsverlaufs und Ausmaß der Änderung, intonatorisches Angepasstsein an das unmittelbar davor und das unmittelbar danach Geäußerte, Tonhöhenregister und Stimmfarbe.

Das intonatorisch sowohl an die unterbrochene Äußerung als auch an ihre Fortsetzung angepasste Anschluss-ja beugt einem vorzeitigen Schließen auf Satzbruch durch den Hörer vor und kündigt eine oder mehrere fehlende Äußerungskonstituenten oder Korrekturen der einzelnen spezifischen Konstituenten an.

Das Anschluss-ja mit einer selbstständigen Intonationskontur kündigt hauptsächlich eine neue Satzkonstruktion an, die sowohl ein neuer Formulierungsversuch nach abgebrochener Vorläuferäußerung als auch einfach eine neue Äußerung nach abgeschlossener Vorläuferäußerung sein kann. Dieses intonatorisch selbstständige Anschluss-ja kann ebenfalls möglichen Äußerungsenden folgen, woran weitere Äußerungskonstituenten angeknüpft werden.

Anschluss-ja mit einer steigend-fallenden oder fallenden Tonhöhenbewegung mit einem Tonhöhensprung nach oben davor scheinen einen Worteinfall zu signalisieren.

\section{Literaturverzeichnis}

Croft, William (2001): Radical Construction Grammar. Syntactic Theory in Typological Perspective. Oxford: Oxford University Press.

Ford, Cecilia E./Thompson, Sandra A. (1996): "Interactional units in conversation: syntactic, intonational, and pragmatic resources for the management of turns". In: Ochs, Elinor et al. (eds.): Interaction and grammar. Cambridge, Cambridge University Press: 134-184.

Hagemann, Jörg (2009): „Tag questions als Evidenzmarker. Formulierungsdynamik, sequentielle Struktur und Funktionen redezuginterner tags". Gesprächsforschung 10: 145176. www.gespraechsforschung-ozs.de [21.04.17].

Indefrey, Peter/Levelt, Willem J. M. (2000): "The neural correlates of language production". In: Michael S. Gazzaniga (ed.): The new cognitive neurosciences. Cambridge etc., MIT Press: $845-865$.

Local, John/Kelly, John (1986): "Projection and 'silences': Notes on phonetic and conversational structure”. Human Studies 9/2: 185-204.

Marić, Dario (2013): „Intonation der mit Fragesätzen verbundenen Sprechhandlungen im Deutschen und Kroatischen“. Zagreber Germanistische Beiträge 22: 71-101.

Meer, Dorothee (2009): „,Unscharfe Ränder’ - Einige kategoriale Überlegungen zu Konstruktionen mit dem Diskursmarker ja in konfrontativen Talkshowpassagen“. In: Günther, Susanne/Bücker, Jörg (eds.): Grammatik im Gespräch. Konstruktionen der Selbst- und Fremdpositionierung. Berlin/New York, de Gruyter: 87-115.

Rost-Roth, Martina (2006): Nachfragen. Formen und Funktionen äußerungsbezogener Interrogationen. Berlin/New York: de Gruyter.

Sacks, Harvey et al. (1974): "A simplest systematic for the organization of turn-taking for conversation". Language 50/4: 696-735.

Schegloff, Emanuel A. (1968): "Sequencing in Conversational Openings". American Anthropologist 70/6: 1075-1095. 
Scheutz, Hannes (1997): „Satzinitiale Voranstellungen im gesprochenen Deutsch als Mittel der Themensteuerung und Referenzkonstitution“. In: Schlobinski, Peter (ed.): Syntax des gesprochenen Deutsch. Opladen, Westdeutscher Verlag: 27-54.

Seghier, L. Mohamed (2013): "The Angular Gyrus: Multiple Functions and Multiple Subdivisions". Neuroscientist 19/1: 43-61.

Selting, Margret (1993): „Voranstellungen vor den Satz. Zur grammatischen Form und interaktiven Funktion von Linksversetzung und Freiem Thema im Deutschen“. Zeitschrift für Germanistische Linguistik 21: 291-319.

Selting, Margret (1995): Prosodie im Gespräch. Aspekte einer interaktionalen Phonologie der Konversation. Tübingen: Niemeyer (= Linguistische Arbeiten 329).

Selting, Margret et al. (2009): „Gesprächsanalytisches Transkriptionssystem 2 (GAT 2)“. Gesprächsforschung 10, 353-402. www.gespraechsforschung-ozs.de [21.04.17].

Sendlmeier, Walter/Seebode, Julia (2006): Formantkarten des deutschen Vokalsystems www.kw.tuberlin.de/fileadmin/a01311100/Formantkarten_des_deutschen_Vokalsystems 01.pdf [21.04.17].

Zifonun, Gisela et al. (1997): Grammatik der deutschen Sprache. Berlin/New York: de Gruyter. 